Nano LIFE

Vol. \#, No. \# (2012) 1-25

(c) World Scientific Publishing Company

\title{
LARGE-SCALE FIRST PRINCIPLES CONFIGURATION INTERACTION CALCULATIONS OF OPTICAL ABSORPTION IN BORON CLUSTERS
}

\author{
RAVINDRA SHINDE \\ Physics Department, Indian Institute of Technology Bombay, \\ Mumbai, Maharashtra 400076, INDIA. \\ ravindra.shinde@iitb.ac.in \\ ALOK SHUKLA \\ Physics Department, Indian Institute of Technology Bombay, \\ Mumbai, Maharashtra 4000\%6, INDIA. \\ shukla@phy.iitb.ac.in
}

Received February 28, 2022

\begin{abstract}
We have performed systematic large-scale all-electron correlated calculations on boron clusters $\mathrm{B}_{n}(\mathrm{n}=2-5)$, to study their linear optical absorption spectra. Several possible isomers of each cluster were considered, and their geometries were optimized at the coupled-cluster singles doubles (CCSD) level of theory. Using the optimized ground-state geometries, excited states of different clusters were computed using the multi-reference singles-doubles configuration-interaction (MRSDCI) approach, which includes electron correlation effects at a sophisticated level. These CI wave functions were used to compute the transition dipole matrix elements connecting the ground and various excited states of different clusters, eventually leading to their linear absorption spectra. The convergence of our results with respect to the basis sets, and the size of the CI expansion were carefully examined. The contribution of configurations to many body wavefunction of various excited states suggests that the excitations involved are collective, plasmonic type.
\end{abstract}

Keywords: photo-absorption, cluster, configuration interaction, boron, optical, MRSDCI

\section{INTRODUCTION}

The area of cluster science has witnessed an enormous progress in terms of both the experimental and theoretical investigations of clusters of various atoms over last few decades. ${ }^{1,2,3,4}$ Ranging from small clusters having a few atoms, to nanotubes, nanosheets, fullerenes etc., clusters have proven their usefulness in the fast emerging field of nanotechnology. ${ }^{5}$ Because of the finiteness of the size, properties of clusters can be greatly tuned, and, therefore they are more amenable to nanoengineer- ing than their bulk counterpart. ${ }^{6}$ The evolution of clusters towards the bulk with the increasing number of atoms, and the underlying mechanism, is a research topic of great contemporary interest. ${ }^{7,8}$

At the present time, boron clusters are attracting great attention because of their novel properties, and potential applications in nanotechnology and hydrogen storage related capabilities..$^{9,10,11,12}$ Boron atom, having $s^{2} p^{1}$ valence electronic configuration, has short covalent radius and tends to form strong directional bonds producing clusters of cova- 
lent nature. Because of this strong covalent bonding, it has hardness close to that of diamond. The ability of boron to form structures of any size due to catenation is only comparable to its neighbor carbon. ${ }^{9}$ Planar boron clusters exhibit aromaticity ${ }^{19}$ due to the presence of itinerant $\pi$ electrons, and some of them are analogous to aromatic hydrocarbons. ${ }^{13}$ Boron fullerenes, boron sheets and singlesheet boron nitride - a graphene analogue - are the other examples of boron-based clusters.

As far as the studies of boron-based clusters are concerned, small ionic boron clusters $\mathrm{B}_{n}^{+}(\mathrm{n} \leqslant$ 20) were experimentally studied by Hanley, Whitten and Anderson. ${ }^{14}$ Wang and coworkers have reported joint theoretical and experimental studies of the electronic structure of bare boron wheels, rings, tubes and large quasi-planar clusters. ${ }^{13,15,16,17}$ Using the photoelectron spectroscopy, they predicted that tubular $\mathrm{B}_{20}$ can act as the smallest boron single walled nanotube. Transition metal-centered boron ionic ring clusters were studied by Constantin et. al. ${ }^{16}$ in a photo-electron spectroscopy experiment, supported by first-principles calculations. The abundance spectrum of boron clusters generated by laser ablation of hexagonal boron nitride was studied by time of flight measurements performed by La Placa, Roland and Wynne. ${ }^{18}$ They also postulated the existence of $\mathrm{B}_{36} \mathrm{~N}_{24}$ cluster having a structure similar to that of $\mathrm{C}_{60}$ fullerene. Lauret et $a l .{ }^{20}$ probed the optical transitions in single walled boron nitride nanotubes by means of optical absorption spectroscopy.

Larger pure boron clusters have also been investigated extensively. Cage-like structure of $\mathrm{B}_{80}-$ similar to $\mathrm{C}_{60}$ fullerene - has been proposed theoretically. ${ }^{21}$ A density functional theory (DFT) study of pure boron sheets and nanotubes was carried out by Cabria, Lopez and Alonso to explore their potential hydrogen storage materials. ${ }^{11}$ Chacko, Kanhere and Boustani investigated different equilibrium geometries of $\mathrm{B}_{24}$ cluster using Born-Oppenheimer molecular dynamics within the framework of DFT. ${ }^{22} \mathrm{Ab}-$ durahman et al. ${ }^{23}$ studied the ladder-like planar boron chains $\mathrm{B}_{n} \quad(n=4-14)$, and computed their static dipole polarizabilities using the $a b$ initio CI method. Johansson discussed strong toroidal ring currents in $\mathrm{B}_{20}$ and other toroidal boron clusters. ${ }^{24}$ Double aromaticity was proposed in toroidal boron clusters $\mathrm{B}_{2 n}(\mathrm{n}=6,14)$ by Bean and Fowler. ${ }^{25}$

Regarding the smaller sized boron clusters, an early theoretical study of boron dimer was carried out by Langhoff and Bauschlicher, ${ }^{26}$ who performed an extensive calculations using the complete-activespace self-consistent-field (CASSCF) multireference configuration interaction (MRCI) with a large basis set. A similar study was carried out by Bruna and Wright ${ }^{27}$ for the excited states of $\mathrm{B}_{2}$, and by Howard and Ray $^{28}$ using the many-body perturbation theory. A systematic geometry and electronic structure calculations of bare boron clusters was reported by Boustani. ${ }^{29}$ He performed all-electron calculations at the SDCI level, but the contracted Gaussian basis sets used were small. Niu, Rao and Jena, ${ }^{30}$ using DFT and quantum chemical methods, presented an account of electronic structures of neutral and charged boron clusters. In their study on small clusters, Möller-Plesset perturbation theory of fourth order (MP4) was used to account for the electron correlation effects. More recently, Atiş, Özdogan, and Güvenç investigated structure and energetics of boron clusters using the DFT. ${ }^{31}$ Aromaticity in planar boron clusters was addressed by Aihara, Kanno and Ishida. ${ }^{32}$

In spite of many theoretical studies of boron clusters of various shapes and sizes, very little experimental information about their ground and excited states is available. Conventional mass spectrometry can distinguish between different clusters only according to their mass, but not according to their geometry. One has to rely on other theoretical or experimental data to be able to differentiate one isomer from another. For example, using first principles calculations of vibrionic fine structure in $\mathrm{C}_{20}^{-}$, and comparing it with experimentally available data, Saito and Y. Miyamoto ${ }^{33}$ identified the cage and bowl structures. Optical absorption spectroscopy, coupled with extensive theoretical calculations of the optical absorption spectra, can be used to distinguish between distinct isomers of clusters produced experimentally, because normally optical absorption spectra are sensitive to the geometries of the clusters. The optical absorption of alkali metal clusters has been extensively studied both experimentally and theoretically. ${ }^{34,35,36,37,38}$ However, a very few such studies exist for the case of boron clusters. Marques and Botti ${ }^{39}$ calculated optical absorption on different $\mathrm{B}_{20}$ isomers using time-dependent (TD) DFT. Boron fullerenes such as $\mathrm{B}_{38}, \mathrm{~B}_{44}, \mathrm{~B}_{80}$ and $\mathrm{B}_{92}$ were also studied by Botti and coworkers ${ }^{40}$ using the same technique. However, to the best of out knowledge, there are no experimental and theoretical study of optical absorption on other bare 
boron clusters, particularly the smaller ones. It is with the aim of filling this void that we undertake a systematic study of the optical absorption in small boron clusters $\mathrm{B}_{n}(\mathrm{n}=2-5)$, employing the MRSDCI method, and high-quality Gaussian basis functions. We perform careful geometry optimization for each possible isomer, and compute the optical absorption spectra of various structures. We also analyze the many-body wave functions of various excited states contributing to the peaks in the computed spectra, and conclude that most of the excitations are collective in nature, signalling the presence of plasmons.

The remainder of this paper is organized as follows. Next section describes the theoretical and computational details of the work, followed by section 3 in which our results are presented and discussed. In section 4 we present our conclusions and discuss possibilities for future work. Detailed information about various excited states contributing to optical absorption is presented in the Appendix.

\section{THEORETICAL AND COMPUTATIONAL DETAILS}

The geometry optimization of various isomers was done using the size-consistent coupled-cluster singles doubles (CCSD) based analytical gradient approach, as implemented in the package GAMESS-Us. ${ }^{41}$ For the purpose, we used the 6$311 \mathrm{G}(\mathrm{d}, \mathrm{p})$ basis set included in the program library ${ }^{41}$ which is known to be well-suited for this task. The process of optimization was initiated by using the geometries reported by Atiş et al., ${ }^{31}$ based upon first principles DFT based calculations. For some simple geometries such as $\mathrm{B}_{2}, \mathrm{~B}_{3}\left(\mathrm{D}_{3 h}\right.$ symmetry), the optimization was carried out manually, by performing the MRSDCI calculations at different geometries, and locating the energy minima. Figure 1 shows the final optimized geometries of the isomers studied in this paper.

Once the ground state geometries of various isomers were determined, the correlated calculations were performed to calculate their low-lying excited states using the multi-reference singlesdoubles configuration-interaction (MRSDCI) approach as implemented in the computer program MELD. ${ }^{42}$ MRSDCI approach is a well-established quantum-chemical approach in which one considers singly- and doubly-excited configurations from a number of reference configurations, leading to a good treatment of electron correlations, both for the ground, and the excited states, in the same calcu- lation. Using the ground- and excited-state wave functions obtained from the MRSDCI calculations, electric dipole matrix elements are computed and subsequently utilized to compute the linear absorption spectrum assuming a Lorentzian line shape. By analyzing the wave functions of the excited states contributing to the peaks of the computed spectrum obtained from a given calculation, bigger MRSDCI calculations were performed by including a larger number of reference states. The choice of the reference states to be included in a given calculation was based upon the magnitude of the corresponding coefficients in the CI wave function of the excited state (or states) contributing to a peak in the spectrum. This procedure was repeated until the computed spectrum converged within an acceptable tolerance, and all the configurations contributing significantly to various excited states were included in the list of the reference states. In the past, we have used such an iterative MRSDCI approach on a number of conjugated polymers to perform large-scale correlated calculations of their linear and nonlinear optical properties. ${ }^{43,44,45,46}$

The number of molecular orbitals, and thus the size of the CI expansion, increases rapidly with the increasing number of atoms in the clusters. Such a proliferation in the size of calculations can essentially make high-quality MRSDCI calculations impossible even for clusters of the sizes discussed in this work. Therefore, wherever possible, we have used the point-group symmetries corresponding to $D_{2 h}$, and its subgroups, at all levels of calculations to reduce the size of the CI expansions. During the MRSDCI calculatiosn, the frozen-core approximation was employed, i.e., while constructing the CI expansion, no virtual excitations from the $1 s$ core electrons of the boron atoms of the cluster were considered. Similarly, excitations into very high energy virtual orbitals were not considered with the purpose of keeping the calculations manageable. The impact of both the frozen-core approximation, and the deletion of high-energy virtual orbitals, along with the influence of the choice of the basis sets on our calculations will be examined carefully in the next section.

\section{RESULTS AND DISCUSSION}

In this section, first we discuss the convergence of our calculations with respect to various approximations and truncation schemes. Thereafter, we present and discuss the results of our calculations 


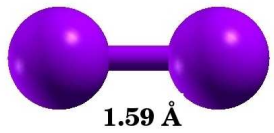

(a) $\mathrm{B}_{2}, \mathrm{D}_{\infty h},{ }^{3} \Sigma_{g}^{-}$

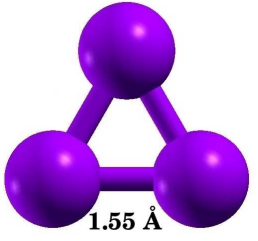

(b) $\mathrm{B}_{3}, \mathrm{D}_{3 h},{ }^{2} \mathrm{~A}_{1}^{\prime}$

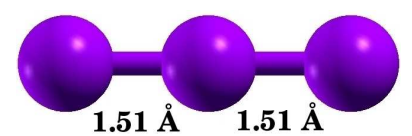

(c) $\mathrm{B}_{3}, \mathrm{D}_{\infty h},{ }^{2} \Sigma_{g}^{-}$

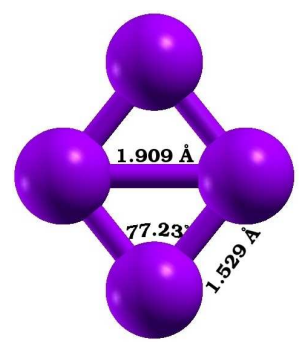

(d) $\mathrm{B}_{4}, \mathrm{D}_{2 h},{ }^{1} \mathrm{~A}_{g}$

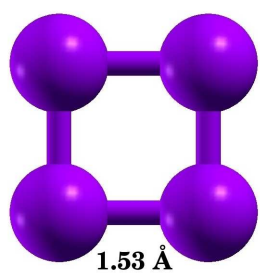

(e) $\mathrm{B}_{4}, \mathrm{D}_{4 h},{ }^{1} \mathrm{~A}_{1 g}$

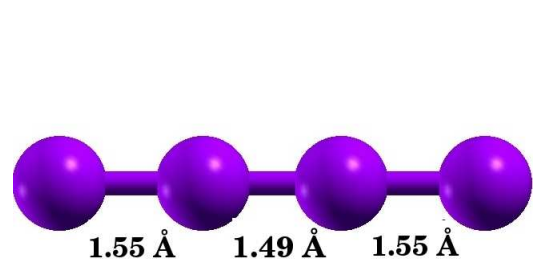

(f) $\mathrm{B}_{4}, \mathrm{D}_{\infty h},{ }^{1} \Sigma_{g}^{-}$

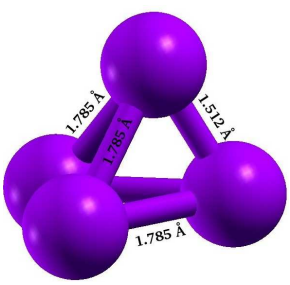

(g) $\mathrm{B}_{4}, \mathrm{C}_{2 v},{ }^{1} \mathrm{~A}_{1}$

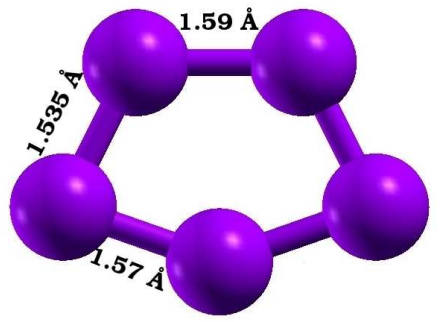

(h) $\mathrm{B}_{5}, \mathrm{C}_{2 v},{ }^{2} \mathrm{~B}_{2}$

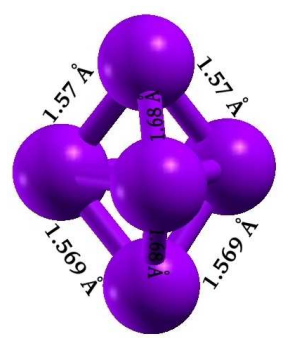

(i) $\mathrm{B}_{5}, \mathrm{C}_{s},{ }^{2} \mathrm{~A}$

Figure 1. (Color online) Geometry optimized structures of boron clusters with point group symmetry and the electronic ground state at the configuration interaction level.

for various clusters.

\subsection{Convergence of Calculations}

Here, we carefully examine the convergence of the calculated absorption spectra with respect to the size and quality of the basis set, along with various truncation schemes in the CI calculations.

\subsubsection{Choice of the basis set}

In general, the results of electronic structure calculations depend upon the quality and the size of the basis set employed. While several contracted Gaussian basis functions have been devised which can deliver high-quality results on various quantities such as the total energy, correlation energy, and the static polarizabilities of molecules, to the best of our knowledge the basis set dependence of linear optical absorption has not been explored. Since boron shows strong covalent bondings, the basis set used for calculations should have diffuse Gaussian contractions. Therefore, to explore the basis set dependence of computed spectra, we used several basis sets ${ }^{49,50}$ to compute the optical absorption spectrum of the smallest cluster, i.e., $\mathrm{B}_{2}$. For the purpose, we used correlation-consistent basis sets named AUG-CC-PVTZ, DAUG-CC-PVDZ, AUG-CC-PVDZ, CC-PVDZ, and DZP, which consist of polarization functions along with diffuse ex-

ponents, and were designed specifically for post Hartree-Fock correlation calculations. ${ }^{49,50}$ From the calculated spectra presented in Fig. 2 the following trends emerge: the spectra computed by various augmented basis sets (AUG-CC-PVTZ, DAUGCC-PVDZ, AUG-CC-PVDZ) are in good agreement with each other in the energy range up to 8 $\mathrm{eV}$, while those obtained using the nonaugmented sets (CC-PVDZ and DZP) disagree with them substantially, particularly in the higher energy range. Given the fact that augmented basis sets are considered superior for molecular calculations, we decided to perform calculations on the all the clusters using the AUG-CC-PVDZ basis set. This is the smallest of the augmented basis sets considered by us, and, therefore, does not cause excessive computational burden when used for larger clusters. 


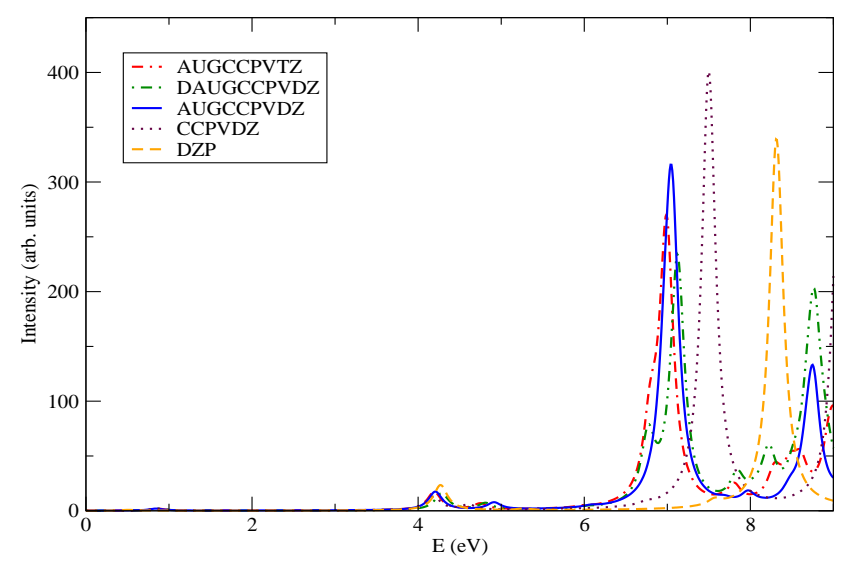

Figure 2. (Color online) Optical absorption in $\mathrm{B}_{2}$ calculated using various Gaussian contracted basis sets. Increasing more and more diffuse $d$ type Gaussians shows negligible effect on optical spectra.

\subsubsection{Orbital Truncation Schemes}

If the total number of orbitals used in a CI expansion is $N$, the number of configurations in the calculation proliferates as $\approx N^{6}$, which can become intractable for large values of $N$. Therefore, it is very important to reduce the number of orbitals used in the CI calculations. The occupied orbitals are reduced by employing the so-called "frozen-core approximation" described earlier, while the unoccupied (virtual) set is reduced by removing very highenergy orbitals.

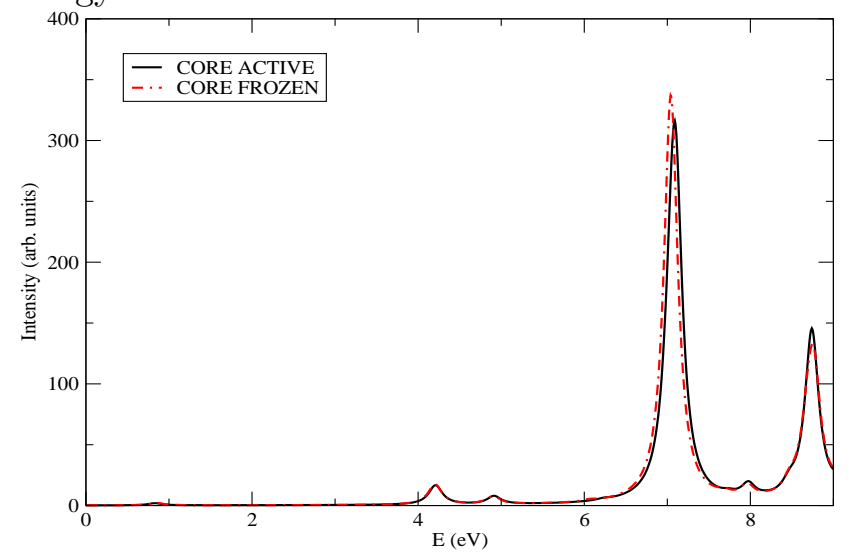

Figure 3. (Color online) The effect of freezing the core orbitals $(1 s)$ of boron atoms on optical absorption spectrum of $\mathrm{B}_{2}$. It renders almost no effect on optical absorption spectrum.

The influence of freezing the $1 s$ core orbitals on the optical absorption spectrum of $\mathrm{B}_{2}$ cluster is displayed in Fig. 3, from which it is obvious that it makes virtually no difference to the results whether or not the core orbitals are frozen. The effect of removing the high-energy virtual orbitals on the ab- sorption spectrum of $\mathrm{B}_{2}$ is examined in Fig. 4. From the figure it is obvious that if all the orbitals above the energy of 1 Hartree are removed, the absorption spectrum stays unaffected. Therefore, in rest of the calculations, wherever needed, orbitals above this energy cutoff were removed from the list of active orbitals. Theoretically speaking this cutoff is sound, because we are looking for absorption features in the energy range much smaller than 1 Hartree.

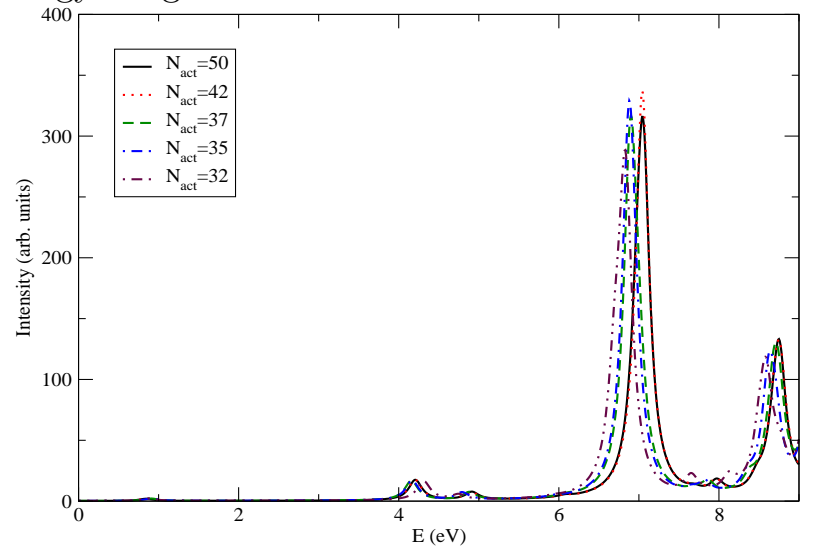

Figure 4. (Color online) The effect of the number of active orbitals $\left(\mathrm{N}_{a c t}\right)$ on the optical absorption spectrum of $\mathrm{B}_{2}$. Until $\mathrm{N}_{a c t}=42$, the optical spectrum does not exhibit any significant change. It corresponds to 1.0 Hartree $(\approx 27.2 \mathrm{eV})$ virtual orbital energy.

\subsubsection{Size of the CI expansion}

As mentioned earlier that the electron correlation effects in both the ground and the excited states were accounted in our calculations by including the relevant configurations in the reference list of the MRSDCI expansion. The greater numerical accuracy demands the inclusion of a large number of configurations in the reference list, but that leads to a rapid growth in the size of the CI expansion, making the calculations numerically prohibitive. However, here we are interested in computing the energy differences rather than the absolute energies of various states, for which good accuracy can be achieved even with moderately large CI expansions. In Table 1 we present the average number of reference states $\left(\mathrm{N}_{r e f}\right)$ included in the MRSDCI expansion and average number of configurations $\left(\mathrm{N}_{\text {total }}\right)$ for different isomers. For a given isomer, the average has been calculated across different irreducible representations which were needed in these symmetry adapted calculations in order to compute the ground and various excited states. The extensiveness of our calculations can be seen from the number $\mathrm{N}_{\text {total }}$, which is $\approx 77000$ for the simplest cluster, and 
around four million for each symmetry subspace of $\mathrm{B}_{5}$. This makes us believe that our results are fairly accurate.

Before we discuss the absorption spectrum for each isomer, we present the ground state energies along with the relative energies of each isomer are given in Table 2. The MRSDCI energy convergence threshold was $10^{-5}$ for all the isomers, with $10^{-4}$ as convegrence threshold for configuration coefficients. From the results it is obvious that as far as the energetics are concerned, for the $\mathrm{B}_{3}$ the triangular structure is most stable, while for $\mathrm{B}_{4}$ and $\mathrm{B}_{5}$ the rhombus and pentagonal structures, respectively, are favorable.

Table 1. The average number of reference configurations $\left(\mathrm{N}_{\text {ref }}\right)$, and average number of total configurations $\left(\mathrm{N}_{\text {total }}\right)$ involved in MRSDCI calculations of various isomers of boron clusters.

\begin{tabular}{cccc}
\hline Cluster & Isomer & $\mathrm{N}_{\text {ref }}$ & $\mathrm{N}_{\text {total }}$ \\
\hline $\mathrm{B}_{2}$ & Linear & 24 & 77245 \\
& & & \\
$\mathrm{~B}_{3}$ & Triangular & 36 & 596798 \\
& Linear & 41 & 671334 \\
& & & \\
$\mathrm{~B}_{4}$ & Rhombus & 37 & 1127918 \\
& Square & 40 & 1070380 \\
& Linear & 34 & 1232803 \\
& Distorted Tetrahedron & 28 & 1253346 \\
$\mathrm{~B}_{5}$ & Pentagon & 22 & 3936612 \\
& Distorted Tri. bipyramid & \\
& & 7 & 3927508 \\
\end{tabular}

Note: ${ }^{a} \mathrm{C}_{s}$ symmetry of isomer converted to $\mathrm{C}_{1}$ in calculations.

Table 2. Ground state (GS) energies (in Hartree) at MRSDCI level and the relative energies (in $\mathrm{eV}$ ) of different isomers of clusters.

\begin{tabular}{cccc}
\hline Cluster & Isomer & $\begin{array}{c}\text { GS energy } \\
(\mathrm{Ha})\end{array}$ & $\begin{array}{c}\text { Relative } \\
\text { energy }(\mathrm{eV})\end{array}$ \\
\hline $\mathrm{B}_{2}$ & Linear & -49.27844 & 0.00 \\
$\mathrm{~B}_{3}$ & Triangular & -73.98998 & 0.00 \\
& Linear & -73.92906 & 1.66 \\
$\mathrm{~B}_{4}$ & Rhombus & -98.74004 & 0.00 \\
& Square & -98.73785 & 0.06 \\
& Linear & -98.66575 & 2.02 \\
& Distorted Tetrahedron & -98.63213 & 2.94 \\
$\mathrm{~B}_{5}$ & Pentagon & -123.42652 & 0.00 \\
& Distorted Tri. Bipyramid & -123.31485 & 3.04 \\
\hline
\end{tabular}

\subsection{Calculated Photoabsorption Spectra of Various Clusters}

Next we present and discuss the results of our photoabsorption calculations for each isomer.

\subsection{1. $\quad B_{2}$}

The simplest and most widely studied cluster of boron is $\mathrm{B}_{2}$ with $\mathrm{D}_{\infty h}$ point group symmetry. Using the SDCI method, we obtained its optimized bond length to be $1.59 \AA$ (cf. Fig. 1(a)), which is in excellent agreement with the experimental value $1.589 \AA . .{ }^{48}$ Using a DFT based methodology, Atiş et al., ${ }^{31}$ obtained a bond length of $1.571 \AA$, while Howard and Ray calculated it to be $1.61 \AA$, using the fourth-order perturbation theory (MP4). ${ }^{28}$

Because the ground state of $\mathrm{B}_{2}$ is a spin triplet, its many-particle wave function predominantly consists of a configuration with two degenerate singlyoccupied molecular orbitals (SOMO) referred to as $H_{1}$ and $H_{2}$ in rest of the discussion. The excited state wave functions will naturally consist of configurations involving electronic excitations from the occupied MOs to the unoccupied MOs starting from lowest unoccupied molecular orbital (LUMOs, $L$ for short). Our calculated photoabsorption spectrum shown in Fig. 5 is characterized by weaker absorptions at low energies, and a very intense one at high energy. The many-particle wave functions of excited states contributing to various peaks are presented in Table 3. A feeble peak appears near $0.85 \mathrm{eV}$, dominated by $\mathrm{H}_{2} \rightarrow L$ and $\mathrm{H}_{2} \rightarrow L+4$ excitations compared to the HF reference configuration. It is followed by a couple of smaller peaks at 4.20 and 4.91 $\mathrm{eV}$. The most intense peak is found at $7.05 \mathrm{eV}$, to which two closely spaced states contribute. Transition to the state near $6.97 \mathrm{eV}$ is polarized transverse to the bond length, while the one close to $7.05 \mathrm{eV}$ carries the bulk of oscillator strength, and is reached by longitudinally polarized photons. All these states exhibit strong mixing of singly-excited configurations. Near $8 \mathrm{eV}$, a smaller peak appears which has strong contributions from doubly-excited configurations $H-1 \rightarrow L ; H_{1} \rightarrow L+2$ and $H-1 \rightarrow L$; $H_{2} \rightarrow L+2$. The wave functions of the excited states contributing to all the peaks exhibit strong configuration mixing, instead of being dominated by single configurations, pointing to the plasmonic nature of the optical excitations. ${ }^{47}$ 


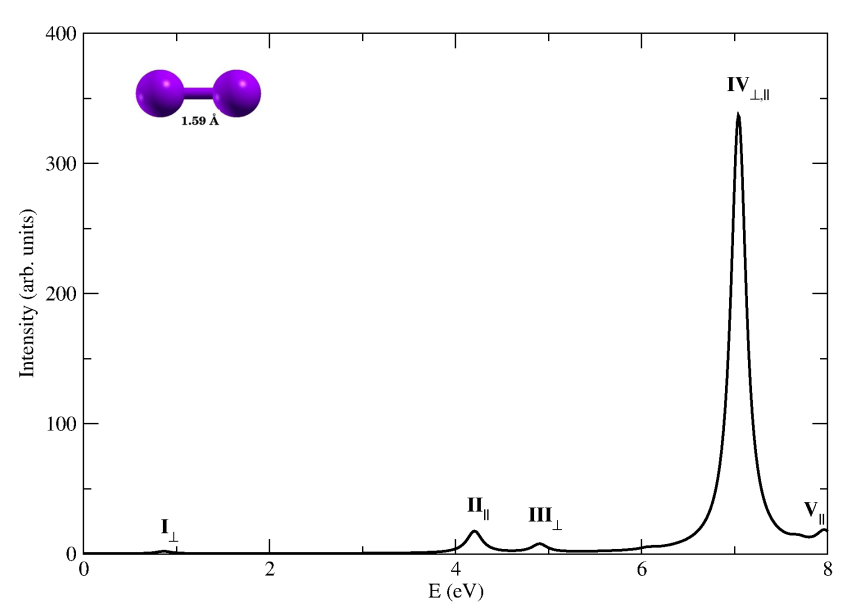

Figure 5. The linear optical absorption spectrum of $\mathrm{B}_{2}$, calculated using the MRSDCI approach. The peaks corresponding to the light polarized along the molecular axis are labeled with the subscript $\|$, while those polarized perpendicular to it are denoted by the subscript $\perp$. For plotting the spectrum, a uniform linewidth of $0.1 \mathrm{eV}$ was used.

\subsection{2. $B_{3}$}

Boron trimer has two possible isomers, triangular and the linear one shown in Figs. 1(b) and 1(c). We found equilateral triangle with $\mathrm{D}_{3 h}$ symmetry to be the most stable isomer. The optimized bond length for triangular isomer is $1.55 \AA$, with the ground state $\left({ }^{2} A_{1}^{\prime}\right)$ energy $1.66 \mathrm{eV}$ lower than that of its linear counterpart. We also explored the possibility of isosceles triangular structure as a favorable one, because $\mathrm{B}_{3}$ is an open-shell system, making it a possible candidate for Jann-Teller distortion. However, the CCSD optimised geometry corresponding to the isosceles structure is so slightly different compared to the equilateral one, that it is unlikely to affect the optical absorption spectrum in a significant manner. Our calculated bond length is in good agreement with experimental value $1.57 \AA,{ }^{14}$ as well as with other reported theoretical values of $1.553 \AA{ }^{29} 1.56$ $\AA^{28}$ and $1.548 \AA^{31}$

The linear $\mathrm{B}_{3}$ isomer with the $\mathrm{D}_{\infty h}$ symmetry, and the ${ }^{2} \Sigma_{g}^{-}$as ground state, was found to have equal bond lengths. Our SDCI optimized bond length of $1.51 \AA$ agrees well with the value $1.518 \AA$ reported by Atiş et al. ${ }^{31}$

The photoabsorption spectra of two isomers of $\mathrm{B}_{3}$ are presented in Figs. 6 and 7. The corresponding many-particle wave functions of excited states contributing to various peaks are presented in Table 5 and 4 . It is obvious that in the linear structure, absorption begins at a lower energy as compared to the triangular one, although the intensity of its low-energy peaks is very small. In the triangular isomer on the other hand, most of the intensity is concentrated at rather high energies, except for a weaker peak close to $3 \mathrm{eV}$. The optical spectra of linear isomer begins with very weak peaks at $0.7 \mathrm{eV}$ (longitudinal polarization) and $2.7 \mathrm{eV}$ (transverse polarization), with their many-particle wave functions dominated by singly-excited configurations. The relatively intense peak at $4.3 \mathrm{eV}$ corresponding to a longitudinally polarized transition, is dominated by doubly-excited configuraitons. It is followed by a small peak mainly due to single excitation $H-2 \rightarrow L$, near $5.9 \mathrm{eV}$. The most intense peak of the spectrum occurs at $7.4 \mathrm{eV}$, followed by another strong peak close to $7.7 \mathrm{eV}$. Both the features correspond to longitudinally polarized transitions, with the many particle wave functions of the concerned states being strong mixtures of single and double excitations with respect to the HF reference state. We note that in the absorption spectrum of the linear cluster, quite expectedly, the bulk of the oscillator strength is carried by longitudinally polarized transitions.

Because the triangular cluster is a planar cluster, its orbitals can be classified as in-plane $\sigma$ orbitals, and the out-of-plane $\pi$ orbitals. Both the HOMO (a singly occupied orbital, in this case) and the LUMO for this isomer are $\sigma$-type orbitals. For this system, two types of optical absorptions are possible: (a) those polarized in the plane of the cluster, and (b) the ones polarized perpendicular to that plane. Our calculations reveal that the transitions correponding to perpendicular polarization $(z$ direction), except for a couple of peaks, have negligible intensities. From Fig. 7 it is obvious that the optical absorption in the triangular isomer starts with a very weak $z$-polarized feature near $0.8 \mathrm{eV}$ (peak I), corresponding to a state with the wave function dominated by single excitations $\left(\pi \rightarrow \sigma^{*}\right)$. This is followed by a series of peaks ranging from II to VI which correpsond to the photons polarized in the plane of the cluster. All these peaks are dominated by states consisting primarily of singly-excited configurations of the $\sigma \rightarrow \sigma^{*}$ type. The most intense peak VI is followed by a shoulder-like feature (VII) corresponding to a $z$-polarized absorption.

If we compare the absorption spectra of the linear and the triangular $\mathrm{B}_{3}$, the peak at $4.34 \mathrm{eV}$ in the spectrum of the linear cluster is the distinguishing feature, and can be used to differentiate between the two isomers. 


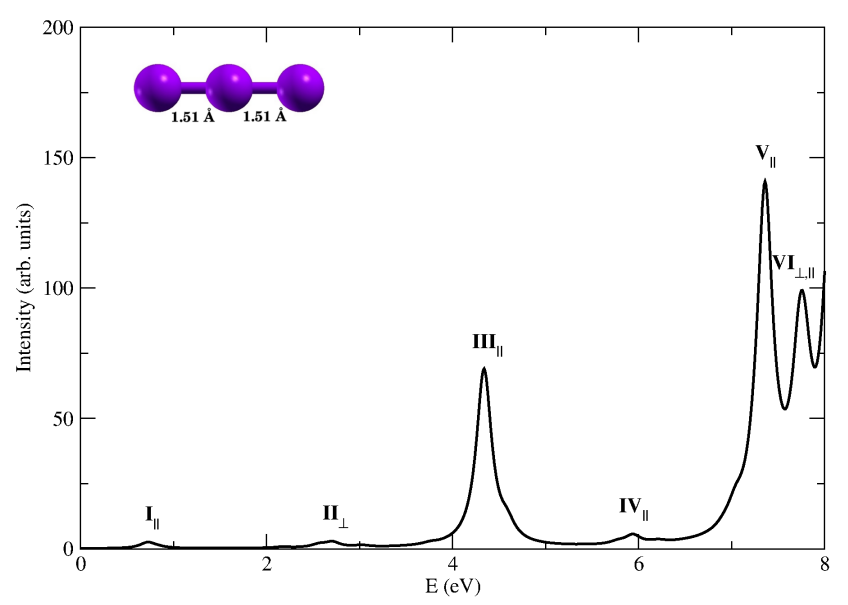

Figure 6. The linear optical absorption spectrum of linear $\mathrm{B}_{3}$, calculated using the MRSDCI approach. The peaks corresponding to the light polarized along the molecular axis are labeled with subscript $\|$, while those polarized perpendicular to it are denoted by the subscript $\perp$. For plotting the spectrum, a uniform linewidth of $0.1 \mathrm{eV}$ was used.

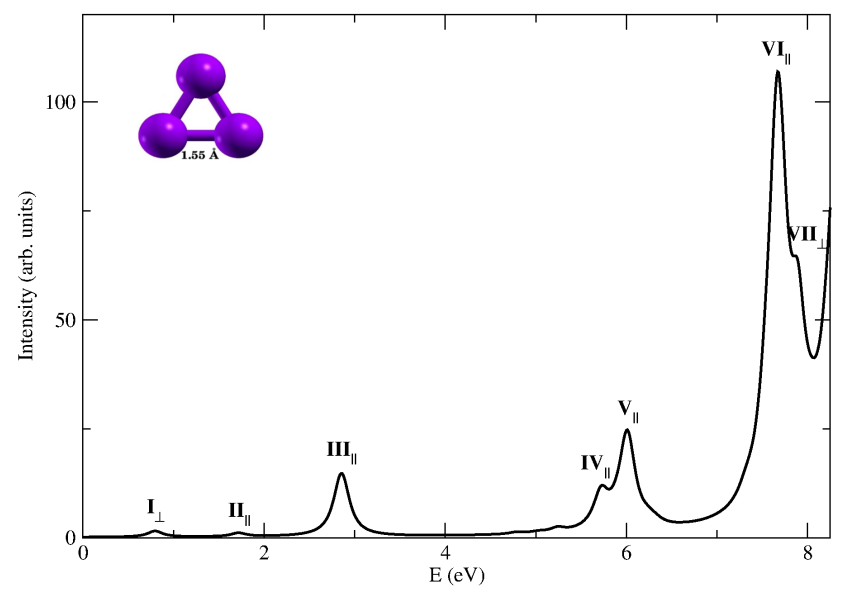

Figure 7. (Color online) The linear optical absorption spectrum of triangular $\mathrm{B}_{3}$ calculated using the MRSDCI approach. Peaks corresponding to light polarized in the plane of the molecule are labeled with subscript $\|$, while those polarized perpendicular to the plane are denoted by the subscript $\perp$. For plotting the spectrum, a uniform linewidth of $0.1 \mathrm{eV}$ was used.

\subsection{3. $B_{4}$}

For the $\mathrm{B}_{4}$ cluster, we investigated the rhombus, square, linear and tetrahedral structures. While the rhombus shaped isomer was found to have the lowest energy, but the square isomer is higher in energy only by a small amount. As a matter of fact, at the HF level the energies of the two isomers were found to be almost degenerate. It was only after the electron correlation effects were included at the CI level that the rhombus stabilized by $\approx 0.06 \mathrm{eV}$ ( $c f$. Table 2)with respect to the square. For the rhom- bus, the ground state was ${ }^{1} A_{g}$, with the optimized bond length $1.529 \AA$, and the short diagonal length $1.909 \AA$. These results are in good agreement with with the correponding lengths of $1.528 \AA$ and 1.885 $\AA$ reported by Boustani, ${ }^{29}$ and $1.523 \AA$ and $1.884 \AA$ computed by Atiş et al. ${ }^{31}$ Both HOMO and LUMO of rhombus isomer are $\sigma$ orbitals.

For the square isomer, with $\mathrm{D}_{4 h}$ symmetry, the electronic ground state is expectedly ${ }^{1} \mathrm{~A}_{1 g}$. As shown in Fig. 1(e), our optimized bound length is $1.53 \AA$, which agrees well with the values $1.527 \AA$ and $1.518 \AA$ as reported in Refs. 29 and 31. In this isomer, HOMO is a $\sigma$ orbital while LUMO is a $\pi$ orbital.

Linear $\mathrm{B}_{4}$, with the $\mathrm{D}_{\infty h}$ symmetry, has the electronic ground state of ${ }^{1} \Sigma_{g}^{-}$. However, energetically linear structure is $2.02 \mathrm{eV}$ higher than the rhombus one ( $c f$. Table 2) which rules out its existence at the room temperatures. As per Fig. 1(f) , the central bond length was found to be $1.49 \AA$, with the two outer bonds being $1.55 \AA$ in length. For the same bonds, Atiş et al. reported these lengths to be $1.487 \AA$ and $1.568 \AA$, respectively. ${ }^{31}$

The distorted tetrahedral structure having $\mathrm{C}_{3 v}$ symmetry, made up of four isosceles triangular faces with lenghts $1.785 \AA, 1.785 \AA$ and $1.512 \AA$. This isomer also lies much higher in energy as compared to the most stable rhombus structure.

The absorption spectra of rhombus, square, linear, and tetrahedral isomers are presented in Figs. $8,9,10$, and 11 respectively. From the figures it is obvious that the general features of the absorption spectra of rhombus and square isomers are similar, except that the rhombus spectrum, with the onset of the absorption near $4 \mathrm{eV}$, is red-shifted by about $1 \mathrm{eV}$ as compared to the square. The absorption spectrum of the linear structure is slightly red-shifted as compared to the rhombus and square shaped isomers, with the majority of absorption occuring in the energy range $5-8 \mathrm{eV}$. This aspect of the photoabsorption in $\mathrm{B}_{4}$ is similar to the case of $\mathrm{B}_{3}$ for which also the linear structure exhibited a redshifted absorption compared to the triangular one.

Since $\mathrm{B}_{4}$ rhombus isomer has $D_{2 h}$ symmetry, we can represent the absorption due to light polarized in different directions in terms of irreducible representations of $D_{2 h}$. So absorption due to in-plane polarized light corresponds to $B_{1 u}$ and $B_{2 u}$, while $B_{3 u}$ corresponds to light polarized in the direction perpendicular to the plane of the isomer. 
The polarization resolved absorption spectrum of rhombus $\mathrm{B}_{4}$, as shown in Fig. 8, exhibits a rather blue-shifted nature as compared to the linear isomer. The many-particle wave functions of excited states contributing to various peaks are presented in Table 6. The onset of spectrum is seen at 4.15 $\mathrm{eV}$ followed by a peak at around $6.12 \mathrm{eV}$. Both of them are due to $y$-polarized component, i.e. along the larger diagonal. The dominant contribution to these peaks come from $\sigma \rightarrow \pi^{*}$ for former, and $\pi \rightarrow \pi^{*}$ for latter. The $x$-component does not contribute much in the whole spectrum, except for minor peaks at $4.2 \mathrm{eV}$ and $6.6 \mathrm{eV}$. It is characterised by mainly $\pi \rightarrow \pi^{*}$ type transitions. It is followed by a relatively low intensity peak at $7.3 \mathrm{eV}$ due to $y$-polarized component with leading contribution from $\sigma \rightarrow \pi^{*}$ transitions. The most intense peak, at $7.84 \mathrm{eV}$, having $y$-polarization component, is characterized by $\sigma \rightarrow \pi^{*}$ type of transitions. There are no direct $H \rightarrow L$ transitions for this isomer, because they are dipole forbidden. The absorption due to light polarized in the direction perpendicular to the plane of isomer is negligible.

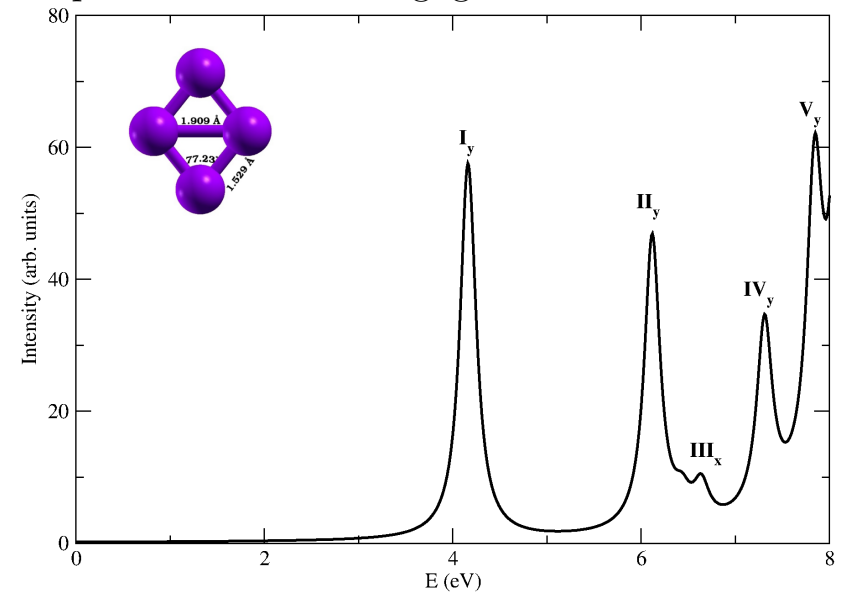

Figure 8. (Color online) The linear optical absorption spectrum of $\mathrm{B}_{4}$ rhombus geometry using the MRSDCI approach. Isomer is aligned in $x-y$ plane with short diagonal along $x$-axis. Peaks corresponding to light polarized along $x$ and $y$-axis are labeled with subscript $x$ and $y$. For plotting the spectrum, a uniform linewidth of $0.1 \mathrm{eV}$ was used.

The square $\mathrm{B}_{4}$ isomer, because of its symmetry, gets equal contribution to absorption spectrum from both $x$ - and $y$-component. It corresponds to in-plane polarization due to $B_{1 u}$ and $B_{2 u}$ irreducible representation, while $B_{3 u}$ corresponds to light polarized in the direction perpendicular to the plane of the isomer. However, in this isomer also, the contribution due to latter is quite negligible. The many-particle wave functions of excited states con- tributing to various peaks are presented in Table 7. It shows just one major peak at $4.88 \mathrm{eV}$ below $7 \mathrm{eV}$, characterized by $\sigma \rightarrow \pi^{*} ; \sigma \rightarrow \pi^{*}$ double excitation. Two smaller peaks appear in this range at $5.5 \mathrm{eV}$ and $6.4 \mathrm{eV}$, with leading contributions from $\sigma \rightarrow \pi^{*} ; \sigma \rightarrow \pi^{*}$ and $\sigma \rightarrow \pi^{*} ; \pi \rightarrow \pi^{*}$ excitations respectively. Beyond $7 \mathrm{eV}$, there are many closely spaced peaks including the most intense one at $7.89 \mathrm{eV}$. It is characterized by double excitation $\sigma \rightarrow \pi^{*} ; \pi \rightarrow \pi^{*}$. In this isomer also, a direct $H \rightarrow L$ transitioin is forbidden. Though, there is very little difference in total energies of rhombus and square isomers of $\mathrm{B}_{4}$, their optical absorption spectra are completely different. They can be easily identified from each other by looking at number of peaks below $7 \mathrm{eV}$ energy. Rhombus exhibits two major peaks, while square has just one.

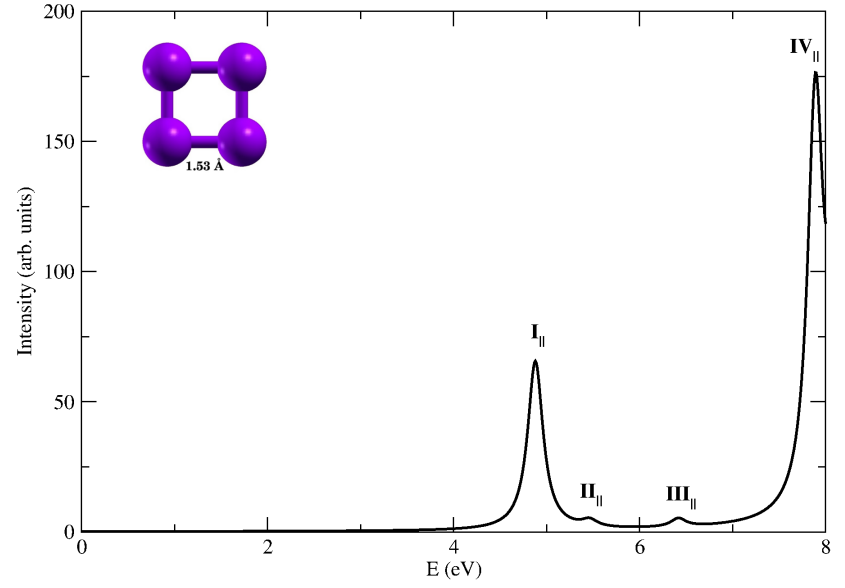

Figure 9. (Color online) The linear optical absorption spectrum of $\mathrm{B}_{4}$ square geometry using MRSDCI approach. Isomer is aligned in $x-y$ plane. Spectrum represents the equal contribution from light polarized in $x$ and $y$ direction. Peaks corresponding to light polarized in the plane of the molecule are labeled with subscript $\|$. For plotting the spectrum, a uniform linewidth of $0.1 \mathrm{eV}$ was used.

Linear $\mathrm{B}_{4}$ isomer exhibits absorption with few, but sharp peaks. The many-particle wave functions of excited states contributing to various peaks are presented in Table 8. The onset of optical absorption occurs near $4.5 \mathrm{eV}$, due to absorption of longaxis polarized light, followed by two major peaks at $5.95 \mathrm{eV}$, and $7.36 \mathrm{eV}$. The first of these two intense peaks, peak II is dominated by singly-excited configurations, while the second one (peak III) is a strong mixture of both singly- and doubly-excited configurations with respect to the HF reference configuration. 


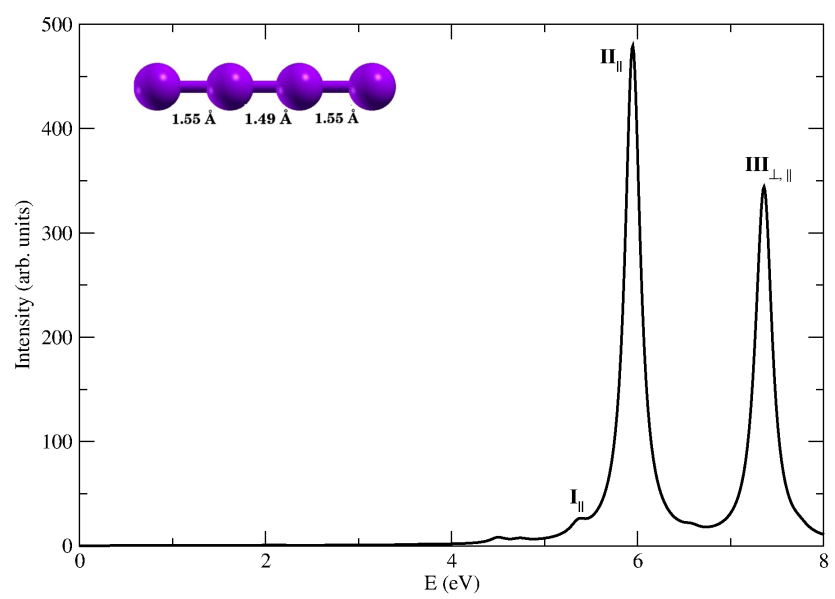

Figure 10. The optical absorption spectrum of linear $\mathrm{B}_{4}$, calculated using the MRSDCI approach. The peaks corresponding to the light polarized along the molecular axis are labeled with subscript $\|$, while those polarized perpendicular to it are denoted by the subscript $\perp$. For plotting the spectrum, a uniform linewidth of $0.1 \mathrm{eV}$ was used.

The 3D structure, a distorted tetrahedral isomer, exhibits an absorption spectrum very different from other isomers, as displayed in Fig. 11. The many-particle wave functions of excited states contributing to various peaks are presented in Table 9. It is the only $\mathrm{B}_{4}$ isomer to exhibit peaks below $4 \mathrm{eV}$. The absorption spectrum is spread over a much larger energy range, and is almost continuous. The oscillator strengths associated with various peaks are much smaller than in other isomers, and most of the peaks appear pairwise. The onset of absorption spectrum is seen at around $1.1 \mathrm{eV}$, characterised mainly by an excited dominated by single-excitation $H \rightarrow L$ ( $c f$. Table 9 ). In this isomer, in contrast to other $\mathrm{B}_{4}$ isomers, direct $H \rightarrow L$ transitions are allowed. Higher energy peaks in this isomer are dominated by doubly-excited configurations, and, are, therefore, sensitive to the electroncorrelation effects.

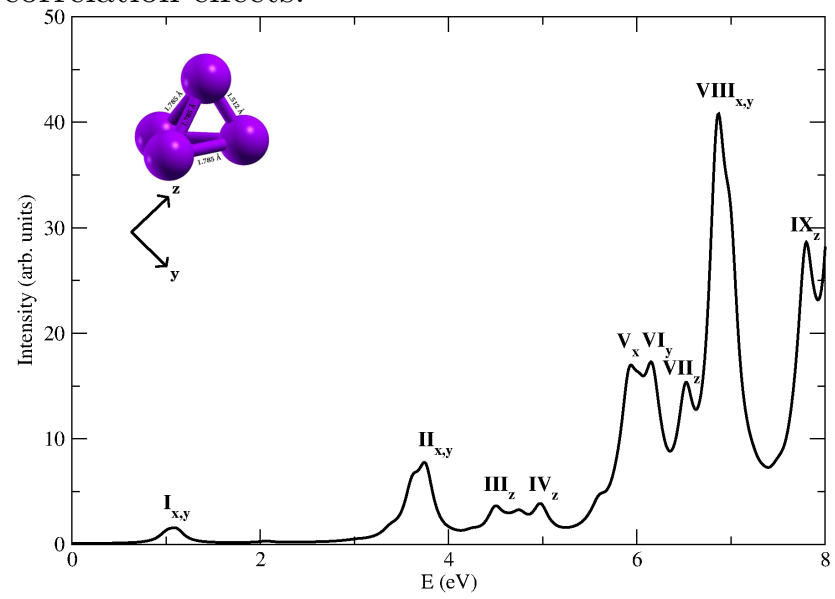

Figure 11. (Color online) The linear optical absorption spectrum of $\mathrm{B}_{4}$ distorted tetrahedral geometry using the MRSDCI approach. Peaks corresponding to light polarized along $x, y$ and $z$-axis are labeled with subscript $x, y$ and $z$ respectively. For plotting the spectrum, a uniform linewidth of $0.1 \mathrm{eV}$ was used.

\subsection{4. $B_{5}$}

We investigated two isomers of $\mathrm{B}_{5}$ : a Jahn-Teller distorted pentagon with the $\mathrm{C}_{2 v}$ symmetry, and (b) a triangular bipyramid with the $\mathrm{C}_{s}$ point group symmetry. The latter one is the second $3-\mathrm{D}$ structure of the boron clusters probed in this work. The lowest lying pentagon isomer, has ${ }^{2} \mathrm{~B}_{2}$ electronic ground state, and is $3.04 \mathrm{eV}$ lower in energy as compared to the bipyramid structure. For the pentagon, the symmetry of ground state at the SCF level was $\mathrm{A}_{1}$, however, at the MRSDCI level the $\mathrm{B}_{2}$ state became lower in energy, in agreement with the previous calculations of Boustani. ${ }^{29}$ Our optimized geometry for the pentagon (Fig. 1(h)) corresponds to an average bond length of $1.56 \AA$, as against 1.57 $\AA$ reported by Boustani, ${ }^{29}$ and $1.644 \AA$ reported by Atiş et $a l .{ }^{31}$ The singly occupied molecular orbital (denoted by $H$ ) and LUMO of pentagon isomers are of $\pi$ and $\sigma$ type, respectiely. The bond lengths for the bipyramid structure are shown in Fig. 1(i), with an average bond length of $1.704 \AA$. The triangular base was found to be isosceles with $1.97 \AA$ as equal sides, and $1.75 \AA$ as the other side.

The absorption spectra of the two isomers are presented in Figs. 12 and 13. The many-particle wave functions of excited states contributing to various peaks are presented in Table 10 and 12 respectively. From the figures it is obvious that the absorption in the bipyramid starts at much lower energies as compared to the pentagonal isomer. Intense absorption peaks in pentagon $\mathrm{B}_{5}$ are located at energies higher than $5 \mathrm{eV}$, with three equally intense peaks at $5.58 \mathrm{eV}, 6.30 \mathrm{eV}$ and $7.16 \mathrm{eV}$, with the photons polarized along the plane of the molecule direction. It has an underlying low intensity absorption contribution from photons polarized along $z-$ direction, which is perpendicular to the molecular plane. The major contribution to the peak at 5.58 $\mathrm{eV}$ comes from $\pi \rightarrow \sigma^{*}$ and $\sigma \rightarrow \sigma^{*}$, single excitations. The latter configuration also contributes to the most intense peak at $6.30 \mathrm{eV}$. The peak at 7.16 $\mathrm{eV}$ is mainly due to $\sigma \rightarrow \sigma^{*}$ type transitions. 


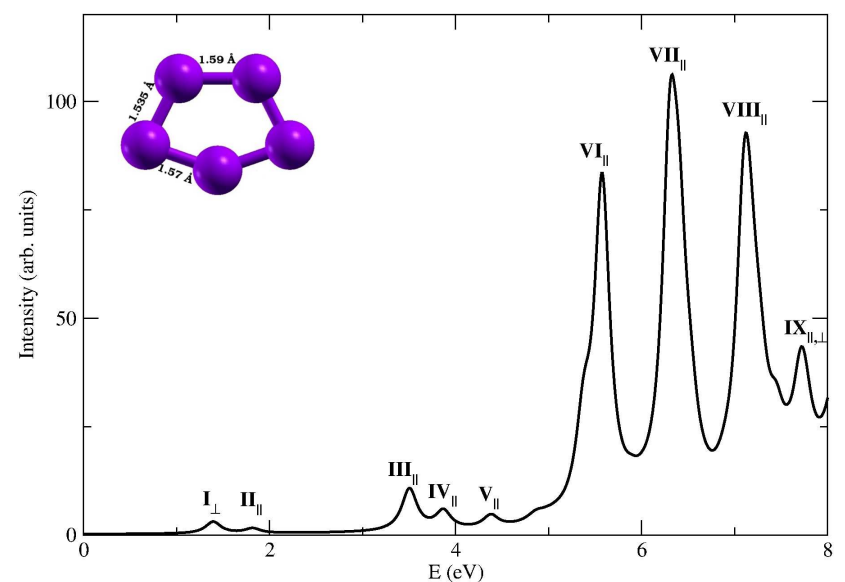

Figure 12. (Color online) The linear photo-absorption spectrum of pentagon $\mathrm{B}_{5}$, calculated using the MRSDCI approach. The peaks corresponding to the light polarized in the plane of the molecule are labeled with subscript $\|$, while those polarized perpendicular to it are denoted by the subscript $\perp$. For plotting the spectrum, a uniform linewidth of $0.1 \mathrm{eV}$ was used.

Since the $\mathrm{B}_{5}$ triagonal bipyramid isomer is not a symmetric one, the calculations were done using $\mathrm{C}_{1}$ symmetry, thereby increasing the difficulty in diagonalizing the Hamiltonian. Hence, in order to reduce the matrix size, we have used a smaller number of reference configurations, and also relaxed the energy convergence threshold criterion a little.

The optical absorption spectrum of $\mathrm{B}_{5}$ triangular bipyramid isomer is exhibited by almost equally spaced peaks at relatively lower energies. The optical absorption starts at $1.74 \mathrm{eV}$ characterized by $H-1 \rightarrow L+4$ configuration. It is followed by two equal intensity peaks at $3.16 \mathrm{eV}$ and $4.27 \mathrm{eV}$ with contributions from single excitations $H-2 \rightarrow$ $H$ and $H-1 \rightarrow L+2$, respectively. The most intense peak is found at $7.52 \mathrm{eV}$ dominated by the doubly excited configuration $H-2 \rightarrow H ; H-1 \rightarrow L+2$. There are two distinguishing features as far as the optical absorption in the two isomers is concerned: (a) presence of three intense peaks in the higher energy region of the absorption spectrum of the pentagonal isomer, and (b) occurance of equally spaced absorption peaks at lower energies in the spectrum of bipyramidal isomer.

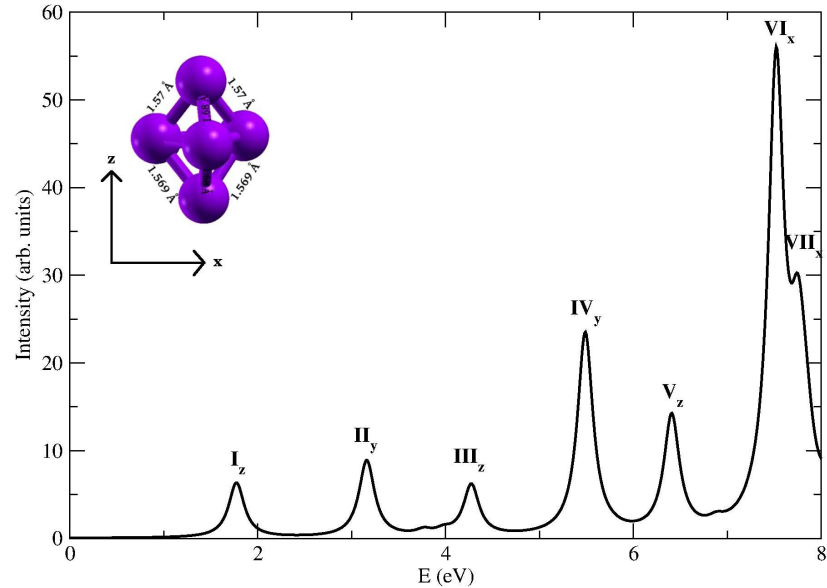

Figure 13. (Color online) The linear photo-absorption spectrum of distorted triangular bipyramid $\mathrm{B}_{5}$, calculated using the MRSDCI approach. Peaks corresponding to light polarized along $x, y$ and $z$-axis are labeled with subscript $x, y$ and $z$ respectively. For plotting the spectrum, a uniform linewidth of $0.1 \mathrm{eV}$ was used.

\section{Conclusions and Outlook}

We presented systematic large-scale all-electron correlated calculations of photoabsorption spectra of boron clusters $\mathrm{B}_{n},(n=2-5)$ with several possible isomers of each cluster. The calculations were perfomred using the MRSDCI method which takes electron correlations into account at a sophisticated level, both for the ground and the excited states. For a cluster consisting of a given number of atoms, significant changes were observed in absorption spectra for different isomers, indicating a strong structure-property relationship. Therefore, our computed spectra can be used in the future photoabsorption experiments to distinguish between different isomers of a cluster, something which is not possible with the conventional mass spectrometry. We also analyzed the many-particle wave functions of various excited states and found them to be a mixture of a large number of configurations, indicating the nature of photoexcited states in these clusters to be plasmonic. ${ }^{47} \mathrm{~A}$ noteworthy aspect of the groundstate photoabsorption of various clusters was the absence of high-intensity peaks in the low-energy region of the spectrum. The most intense peaks occurred at higher energies involving orbitals away from the Fermi level, consistent with the fact that the bulk boron is an indirect bandgap semiconductor, with no optical absorption at the gap. In other words, optical absorption features of bulk boron were already evident in smaller clusters. Given the fact that aluminum has the same valence 
shell structure as boron, it will be interesting to perform a similar set of calculations on small aluminum clusters, in order to compare and contrast their photophysics. Calculations along those directions are presently underway in our group, and results will be communicated in future publications.

\section{Acknowledgments}

R. S. thanks the Council of Scientific and Industrial Research (CSIR), India, for a Junior Research Fellowship.

\section{Appendix: Excited State CI Wavefunc- tions, Energies and Transition Moments}

In the following tables we have given the excitation energies (with respect to the ground state), the many body wavefunction and the oscillator strengths of the excited states corresponding to the peaks in the photoabsorption spectra of various isomers listed in Fig. 1, and discussed in section 3. 
Table 3. Excitation energies, $E$, and many-particle wave functions of excited states corresponding to the peaks in the linear absorption spectrum of $\mathrm{B}_{2}$ (cf. Fig. 5), along with the oscillator strength of the transitions $\left(f_{12}=\frac{2}{3} \frac{m_{e}}{\hbar^{2}}\left(E_{2}-E_{1}\right) \sum_{i}\left|\left\langle m\left|d_{i}\right| G\right\rangle\right|^{2}\right)$, where, $|m\rangle$ denotes the excited state in question, $|G\rangle$, the ground state, and $d_{i}$ is the $i$-th Cartesian component of the electric dipole operator. Longitudinal and transverse polarization corresponds to the absorption due to light polarized along and perpendicular to the molecular axis respectively. In the wave function, the bracketed numbers are the CI coefficients of a given electronic configuration. Symbols $H_{1}, H_{2}$ denote SOMOs discussed earlier, and $H$, and $L$, denote HOMO and LUMO orbitals respectively. $H F$ denotes the Hartree-Fock configuration.

\begin{tabular}{|c|c|c|c|c|}
\hline Peak & $E(\mathrm{eV})$ & $f_{12}$ & Polarization & Wave Function \\
\hline $\mathrm{GS}^{a}$ & & & & $\begin{array}{l}|H F\rangle(0.8673) \\
|H-1 \rightarrow L ; H-1 \rightarrow L\rangle(0.2706) \\
|H-1 \rightarrow L ; H-1 \rightarrow L+4\rangle(0.1283)\end{array}$ \\
\hline I & 0.845 & 0.1187 & transverse & $\begin{array}{l}\left|H_{2} \rightarrow L\right\rangle(0.8742) \\
\left|H_{2} \rightarrow L+4\right\rangle(0.2194)\end{array}$ \\
\hline II & 4.207 & 1.1449 & longitudinal & $\begin{array}{l}|H-1 \rightarrow L\rangle(0.7599) \\
\left|H_{1} \rightarrow L+7\right\rangle(0.2873) \\
\left|H_{2} \rightarrow L+7\right\rangle(0.2873)\end{array}$ \\
\hline III & 4.914 & 0.4252 & transverse & $\begin{array}{l}|H-1 \rightarrow L ; H-1 \rightarrow L+2\rangle(0.7553) \\
|H-2 \rightarrow L+2\rangle(0.3186) \\
|H-1 \rightarrow L+7\rangle(0.266)\end{array}$ \\
\hline IV & $\begin{array}{l}6.97 \\
7.05\end{array}$ & $\begin{array}{r}4.3060 \\
18.0923\end{array}$ & $\begin{array}{l}\text { transverse } \\
\text { longitudinal }\end{array}$ & $\begin{array}{l}\left|H_{2} \rightarrow L+4\right\rangle(0.5600) \\
\left|H_{2} \rightarrow L+5\right\rangle(0.5150) \\
|H-1 \rightarrow L\rangle(0.4462) \\
\left|H_{2} \rightarrow L+3\right\rangle(0.3346) \\
\left|H_{1} \rightarrow L+3\right\rangle(0.3346) \\
|H-1 \rightarrow L+4\rangle(0.2921)\end{array}$ \\
\hline $\mathrm{V}$ & 7.973 & 0.6907 & longitudinal & $\begin{array}{l}\left|H-1 \rightarrow L ; H_{2} \rightarrow L+2\right\rangle(0.5613) \\
\left|H-1 \rightarrow L ; H_{1} \rightarrow L+2\right\rangle(0.5613) \\
|H-1 \rightarrow L+4\rangle(0.2303)\end{array}$ \\
\hline
\end{tabular}

Note: ${ }^{a}$ GS does not correspond to any peak, instead it corresponds to the ground state wavefunction of the isomer. 
Table 4. Excitation energies, $E$, and many-particle wave functions of excited states corresponding to the peaks in the linear absorption spectrum of triangular $\mathrm{B}_{3}$ ( $c f$. Fig. 7), along with oscillator strength of transition $\left(f_{12}=\frac{2}{3} \frac{m_{e}}{\hbar^{2}}\left(E_{2}-E_{1}\right) \sum_{i}\left|\left\langle m\left|d_{i}\right| G\right\rangle\right|^{2}\right)$, where, $|m\rangle$ denotes the excited state in question, $|G\rangle$, the ground state, and $d_{i}$ is the $i$-th Cartesian component of the electric dipole operator. The polarization || corresponds to the absorption due to light polarized in the plane of isomer, while $\perp$ corresponds to the polarization perpendicular to that plane. In the wave function, the bracketed numbers are the CI coefficients of a given electronic configuration. Symbol $L$ and $H$ denote LUMO and SOMO orbitals discussed earlier. $H F$ denotes the Hartree-Fock configuration.

\begin{tabular}{|c|c|c|c|c|}
\hline Peak & $E(\mathrm{eV})$ & $f_{12}$ & Polarization & Wave Function \\
\hline $\mathrm{GS}^{a}$ & & & & $\begin{array}{l}|H F\rangle(0.8229) \\
|H-3 \rightarrow L\rangle(0.2162) \\
|H \rightarrow L\rangle(0.2072) \\
|H-3 \rightarrow H\rangle(0.1544)\end{array}$ \\
\hline $\mathrm{I}$ & 0.797 & 0.0912 & $\perp$ & $\begin{array}{l}|H-1 \rightarrow H\rangle(0.7357) \\
|H-1 \rightarrow L\rangle(0.4693) \\
|H-1 \rightarrow L+8\rangle(0.1142)\end{array}$ \\
\hline II & 1.710 & 0.0282 & $\|$ & $\begin{array}{l}|H \rightarrow L+1\rangle(0.4816) \\
|H-2 \rightarrow H\rangle(0.4315) \\
|H-2 \rightarrow L\rangle(0.3757) \\
|H-3 \rightarrow L+1\rangle(0.3709) \\
|H \rightarrow L\rangle(0.5041) \\
|H-3 \rightarrow H\rangle(0.4703)\end{array}$ \\
\hline III & 2.872 & 0.5173 & $\|$ & $\begin{array}{l}|H-2 \rightarrow H\rangle(0.5374) \\
|H \rightarrow L+1\rangle(0.5199) \\
|H-2 \rightarrow L\rangle(0.3306) \\
|H-3 \rightarrow L+1\rangle(0.2216) \\
|H \rightarrow L\rangle(0.4906) \\
|H-3 \rightarrow L\rangle(0.4499)\end{array}$ \\
\hline IV & $\begin{array}{l}5.710 \\
5.730\end{array}$ & $\begin{array}{l}0.3259 \\
0.2053\end{array}$ & $\|$ & $\begin{array}{l}|H-2 \rightarrow L\rangle(0.4114) \\
|H-2 \rightarrow H\rangle(0.3474) \\
|H-3 \rightarrow L\rangle(0.4202) \\
|H-2 \rightarrow H ; H-3 \rightarrow L\rangle(0.2050)\end{array}$ \\
\hline $\mathrm{V}$ & 5.988 & 0.9679 & $\|$ & $\begin{array}{l}|H-3 \rightarrow L+1\rangle(0.3968) \\
|H-2 \rightarrow L\rangle(0.3057) \\
|H-2 \rightarrow H\rangle(0.1578) \\
|H-3 \rightarrow L\rangle(0.2578) \\
|H \rightarrow L\rangle(0.1908)\end{array}$ \\
\hline VI & 7.657 & 1.6393 & $\|$ & $\begin{array}{l}|H \rightarrow L+2\rangle(0.5863) \\
|H-3 \rightarrow L+2\rangle(0.2497) \\
|H \rightarrow L+14\rangle(0.2431) \\
|H \rightarrow L+4\rangle(0.2093) \\
|H \rightarrow L+3\rangle(0.2991) \\
|H-3 \rightarrow L+1\rangle(0.2648) \\
|H-2 \rightarrow H\rangle(0.1794) \\
|H-2 \rightarrow L\rangle(0.1521)\end{array}$ \\
\hline VII & 7.893 & 2.1315 & $\perp$ & $\begin{array}{l}|H \rightarrow L+5\rangle(0.7611) \\
|H-3 \rightarrow L+4\rangle(0.3477)\end{array}$ \\
\hline
\end{tabular}

Note: ${ }^{a}$ GS does not correspond to any peak, instead it corresponds to the ground state wavefunction of the isomer. 
Table 5. Excitation energies, $E$, and many-particle wave functions of excited states corresponding to the peaks in the linear absorption spectrum of linear $\mathrm{B}_{3}$ ( $c f$. Fig. 6), along with oscillator strength of transition $\left(f_{12}=\frac{2}{3} \frac{m_{e}}{\hbar^{2}}\left(E_{2}-E_{1}\right) \sum_{i}\left|\left\langle m\left|d_{i}\right| G\right\rangle\right|^{2}\right)$, where, $|m\rangle$ denotes the excited state in question, $|G\rangle$, the ground state, and $d_{i}$ is the $i$-th Cartesian component of the electric dipole operator. Longitudinal and transverse polarization corresponds to the absorption due to light polarized along and perpendicular to the molecular axis respectively. In the wave function, the bracketed numbers are the CI coefficients of a given electronic configuration. Symbols $H$ and $L$ denote HOMO and LUMO orbitals respectively, and $H_{1}$ denotes SOMOs discussed earlier. $H F$ denotes the Hartree-Fock configuration.

\begin{tabular}{|c|c|c|c|c|}
\hline Peak & $E(\mathrm{eV})$ & $f_{12}$ & Polarization & Wave Function \\
\hline $\mathrm{GS}^{a}$ & & & & $\begin{array}{l}|H F\rangle(0.6650) \\
|H-1 \rightarrow L ; H-1 \rightarrow L\rangle(0.3286) \\
|H-1 \rightarrow L ; H-1 \rightarrow L\rangle(0.3277) \\
|H \rightarrow L ; H-1 \rightarrow L+1\rangle(0.2158) \\
|H \rightarrow L ; H-1 \rightarrow L+1\rangle(0.2157)\end{array}$ \\
\hline I & 0.723 & 0.1520 & longitudinal & $|H \rightarrow L\rangle(0.8222)$ \\
\hline II & 2.707 & 0.1337 & transverse & $|H-1 \rightarrow L\rangle(0.5360)$ \\
\hline III & 4.338 & 4.5187 & longitudinal & $\begin{array}{l}|H-1 \rightarrow L ; H-1 \rightarrow H\rangle(0.5826) \\
|H-1 \rightarrow L ; H-1 \rightarrow H\rangle(0.5826)\end{array}$ \\
\hline IV & 5.937 & 0.2295 & longitudinal & $|H-2 \rightarrow L\rangle(0.2497)$ \\
\hline $\mathrm{V}$ & 7.359 & 8.4221 & longitudinal & $\begin{array}{l}|H-1 \rightarrow L+1\rangle(0.3683) \\
|H-1 \rightarrow L+1\rangle(0.3683) \\
|H-3 \rightarrow L ; H \rightarrow L\rangle(0.3301) \\
|H-3 \rightarrow H\rangle(0.2364)\end{array}$ \\
\hline VI & 7.786 & 2.3331 & transverse & $\begin{array}{l}|H-2 \rightarrow L\rangle(0.3858) \\
|H \rightarrow L ; H-1 \rightarrow L+4\rangle(0.2851) \\
|H \rightarrow L ; H-1 \rightarrow L+4\rangle(0.2851) \\
|H-1 \rightarrow L ; H-1 \rightarrow L+4\rangle(0.6438) \\
|H-1 \rightarrow L ; H-1 \rightarrow L+4\rangle(0.3905)\end{array}$ \\
\hline
\end{tabular}

Note: ${ }^{a}$ GS does not correspond to any peak, instead it corresponds to the ground state wavefunction of the isomer. 
Table 6. Excitation energies, $E$, and many-particle wave functions of excited states corresponding to the peaks in the linear absorption spectrum of rhombus $\mathrm{B}_{4}$ ( $c f$. Fig. 8), along with oscillator strength of transition $\left(f_{12}=\frac{2}{3} \frac{m_{e}}{\hbar^{2}}\left(E_{2}-E_{1}\right) \sum_{i}\left|\left\langle m\left|d_{i}\right| G\right\rangle\right|^{2}\right)$, where, $|m\rangle$ denotes the excited state in question, $|G\rangle$, the ground state, and $d_{i}$ is the $i$-th Cartesian component of the electric dipole operator. The polarization in-plane corresponds to the absorption due to light polarized in the plane of isomer. In the wave function, the bracketed numbers are the CI coefficients of a given electronic configuration. Symbols $H / L$ denote HOMO/LUMO orbitals. $H F$ denotes the Hartree-Fock configuration.

\begin{tabular}{|c|c|c|c|c|}
\hline Peak & $E(\mathrm{eV})$ & $f_{12}$ & Polarization & Wave Function \\
\hline $\mathrm{GS}^{a}$ & & & & $\begin{array}{l}|H F\rangle(0.8787) \\
|H \rightarrow L ; H \rightarrow L\rangle(0.1147)\end{array}$ \\
\hline I & 4.159 & 3.5758 & in-plane & $\begin{array}{l}|H \rightarrow L+2\rangle(0.6566) \\
|H \rightarrow L+10\rangle(0.3210) \\
|H-1 \rightarrow L+6\rangle(0.2773) \\
|H-1 \rightarrow L+17\rangle(0.1850)\end{array}$ \\
\hline II & 6.118 & 2.9911 & in-plane & $\begin{array}{l}|H-1 \rightarrow L+6\rangle(0.5786) \\
|H-1 \rightarrow L+17\rangle(0.3285) \\
|H-3 \rightarrow L\rangle(0.2656) \\
|H-2 \rightarrow L+11\rangle(0.2544) \\
|H-2 \rightarrow L+18\rangle(0.2492)\end{array}$ \\
\hline III & 6.639 & 0.3735 & in-plane & $\begin{array}{l}|H-1 \rightarrow L+3\rangle(0.4496) \\
|H-1 \rightarrow L+13\rangle(0.4485) \\
|H-4 \rightarrow L\rangle(0.4052) \\
|H \rightarrow L+11\rangle(0.2766) \\
|H \rightarrow L+1\rangle(0.2329) \\
|H \rightarrow L+18\rangle(0.1905)\end{array}$ \\
\hline IV & 7.311 & 1.9267 & in-plane & $\begin{array}{l}|H \rightarrow L+2\rangle(0.3055) \\
|H-2 \rightarrow L+11\rangle(0.2892) \\
|H-3 \rightarrow L\rangle(0.2834) \\
|H-2 \rightarrow L+18\rangle(0.2487) \\
|H-2 \rightarrow L+1\rangle(0.2029) \\
|H-2 \rightarrow L+5\rangle(0.1509)\end{array}$ \\
\hline $\mathrm{V}$ & 7.842 & 3.0105 & in-plane & $\begin{array}{l}|H \rightarrow L+2\rangle(0.4233) \\
|H \rightarrow L+10\rangle(0.3270) \\
|H-2 \rightarrow L+11\rangle(0.2049) \\
|H \rightarrow L+20\rangle(0.1946) \\
|H-2 \rightarrow L+1\rangle(0.1594) \\
|H-2 \rightarrow L+18\rangle(0.1582)\end{array}$ \\
\hline
\end{tabular}

Note: ${ }^{a}$ GS does not correspond to any peak, instead it corresponds to the ground state wavefunction of the isomer. 
Table 7. Excitation energies, $E$, and many-particle wave functions of excited states corresponding to the peaks in the linear absorption spectrum of square $\mathrm{B}_{4}$ ( $c f$. Fig. 9), along with oscillator strength of transition $\left(f_{12}=\frac{2}{3} \frac{m_{e}}{\hbar^{2}}\left(E_{2}-E_{1}\right) \sum_{i}\left|\left\langle m\left|d_{i}\right| G\right\rangle\right|^{2}\right)$, where, $|m\rangle$ denotes the excited state in question, $|G\rangle$, the ground state, and $d_{i}$ is the $i$-th Cartesian component of the electric dipole operator. The polarization in-plane corresponds to the absorption due to light polarized in the plane of isomer. In the wave function, the bracketed numbers are the CI coefficients of a given electronic configuration. Symbols $H / L$ denote HOMO/LUMO orbitals. $H F$ denotes the Hartree-Fock configuration.

\begin{tabular}{|c|c|c|c|c|}
\hline Peak & $E(\mathrm{eV})$ & $f_{12}$ & Polarization & Wave Function \\
\hline $\mathrm{GS}^{a}$ & & & & $\begin{array}{l}|H F\rangle(0.8682) \\
|H \rightarrow L ; H \rightarrow L\rangle(0.1765) \\
|H-2 \rightarrow L ; H-2 \rightarrow L\rangle(0.0920)\end{array}$ \\
\hline I & 4.879 & 4.3269 & in-plane & $\begin{array}{l}|H \rightarrow L ; H \rightarrow L+1\rangle(0.2946) \\
|H \rightarrow L ; H-3 \rightarrow L\rangle(0.1909) \\
|H-2 \rightarrow L ; H-3 \rightarrow L\rangle(0.1651)\end{array}$ \\
\hline II & 5.462 & 0.1792 & in-plane & $\begin{array}{l}|H \rightarrow L ; H \rightarrow L+1\rangle(0.5893) \\
|H \rightarrow L ; H \rightarrow L+6\rangle(0.2477)\end{array}$ \\
\hline III & 6.418 & 0.2165 & in-plane & $\begin{array}{l}|H \rightarrow L ; H-3 \rightarrow L\rangle(0.5379) \\
|H \rightarrow L ; H \rightarrow L+1\rangle(0.2922) \\
|H-2 \rightarrow L ; H-3 \rightarrow L\rangle(0.1744)\end{array}$ \\
\hline IV & 7.890 & 10.4628 & in-plane & $|H \rightarrow L+1 ; H-1 \rightarrow L\rangle(0.2351)$ \\
\hline
\end{tabular}

Note: ${ }^{a}$ GS does not correspond to any peak, instead it corresponds to the ground state wavefunction of the isomer. 
Table 8. Excitation energies, $E$, and many-particle wave functions of excited states corresponding to the peaks in the linear absorption spectrum of linear $\mathrm{B}_{4}$ ( $c f$. Fig. 10), along with oscillator strength of transition $\left(f_{12}=\frac{2}{3} \frac{m_{e}}{\hbar^{2}}\left(E_{2}-E_{1}\right) \sum_{i}\left|\left\langle m\left|d_{i}\right| G\right\rangle\right|^{2}\right)$, where, $|m\rangle$ denotes the excited state in question, $|G\rangle$, the ground state, and $d_{i}$ is the $i$-th Cartesian component of the electric dipole operator. Longitudinal and transverse polarization corresponds to the absorption due to light polarized along and perpendicular to the molecular axis respectively. In the wave function, the bracketed numbers are the CI coefficients of a given electronic configuration. Symbols $H / L$ denote HOMO/LUMO orbitals. $H F$ denotes the Hartree-Fock configuration.

\begin{tabular}{|c|c|c|c|c|}
\hline Peak & $E(\mathrm{eV})$ & $f_{12}$ & Polarization & Wave Function \\
\hline $\mathrm{GS}^{a}$ & & & & $\begin{array}{l}|H F\rangle(0.5636) \\
|H \rightarrow L\rangle(0.4737) \\
|H-1 \rightarrow L ; H \rightarrow L+1\rangle(0.2291) \\
|H-1 \rightarrow L ; H \rightarrow L+1\rangle(0.2289)\end{array}$ \\
\hline I & 5.363 & 0.7433 & longitudinal & $\begin{array}{l}|H \rightarrow L ; H-1 \rightarrow L+9\rangle(0.3005) \\
|H \rightarrow L ; H-1 \rightarrow L+9\rangle(0.3005) \\
|H \rightarrow L ; H-1 \rightarrow L+8\rangle(0.2702) \\
|H \rightarrow L ; H-1 \rightarrow L+8\rangle(0.2702) \\
|H \rightarrow L ; H-1 \rightarrow L+4\rangle(0.2439) \\
|H \rightarrow L ; H-1 \rightarrow L+4\rangle(0.2439)\end{array}$ \\
\hline II & 5.947 & 31.8150 & longitudinal & $\begin{array}{l}|H-1 \rightarrow L+1\rangle(0.2957) \\
|H-1 \rightarrow L+1\rangle(0.2957)\end{array}$ \\
\hline III & 7.352 & 14.0546 & longitudinal & $\begin{array}{l}|H \rightarrow L ; H-2 \rightarrow L\rangle(0.6477) \\
|H-3 \rightarrow L\rangle(0.2966) \\
|H-1 \rightarrow L+1\rangle(0.2091) \\
|H-1 \rightarrow L+1\rangle(0.2091) \\
|H-1 \rightarrow L+13\rangle(0.5253)\end{array}$ \\
\hline
\end{tabular}

Note: ${ }^{a}$ GS does not correspond to any peak, instead it corresponds to the ground state wavefunction of the isomer. 
Table 9. Excitation energies, $E$, and many-particle wave functions of excited states corresponding to the peaks in the linear absorption spectrum of distorted tetrahedron $\mathrm{B}_{4}$ ( $c f$. Fig. 11), along with oscillator strength of transition $\left(f_{12}=\frac{2}{3} \frac{m_{e}}{\hbar^{2}}\left(E_{2}-E_{1}\right) \sum_{i}\left|\left\langle m\left|d_{i}\right| G\right\rangle\right|^{2}\right)$, where, $|m\rangle$ denotes the excited state in question, $|G\rangle$, the ground state, and $d_{i}$ is the $i$-th Cartesian component of the electric dipole operator. The polarization $x, y$ and $z$ corresponds to the absorption due to light polarized along $x-, y-$ and $z$ - axis respectively. In the wave function, the bracketed numbers are the CI coefficients of a given electronic configuration. Symbols $H / L$ denote HOMO/LUMO orbitals. $H F$ denotes the Hartree-Fock configuration.

\begin{tabular}{|c|c|c|c|c|}
\hline Peak & $E(\mathrm{eV})$ & $f_{12}$ & Polarization & Wave Function \\
\hline $\mathrm{GS}^{a}$ & & & & $\begin{array}{l}|H F\rangle(0.6493) \\
|H \rightarrow L ; H \rightarrow L+2\rangle(0.4695) \\
|H \rightarrow L ; H-3 \rightarrow L+2\rangle(0.1547)\end{array}$ \\
\hline I & 1.000 & 0.0517 & $\mathrm{x}$ & $\begin{array}{l}|H \rightarrow L\rangle(0.8127) \\
|H \rightarrow L ; H-1 \rightarrow L+2\rangle(0.2364)\end{array}$ \\
\hline & 1.111 & 0.0714 & $\mathrm{y}$ & $\begin{array}{l}|H \rightarrow L+2 ; H \rightarrow L+1\rangle(0.5944) \\
|H \rightarrow L ; H \rightarrow L+1\rangle(0.5121)\end{array}$ \\
\hline II & 3.754 & 0.3957 & $\mathrm{x}$ & $\begin{array}{l}|H \rightarrow L ; H-1 \rightarrow L+2\rangle(0.5094) \\
|H \rightarrow L ; H-1 \rightarrow L\rangle(0.4535) \\
|H \rightarrow L+2\rangle(0.3480) \\
|H \rightarrow L+1 ; H \rightarrow L+2\rangle(0.2391)\end{array}$ \\
\hline III & 4.48 & 0.4818 & $\mathrm{z}$ & $|H-1 \rightarrow L\rangle(0.4418)$ \\
\hline IV & 4.96 & 0.5841 & $\mathrm{z}$ & $\begin{array}{l}|H-1 \rightarrow L\rangle(0.2678) \\
|H \rightarrow L ; H-3 \rightarrow L\rangle(0.2227)\end{array}$ \\
\hline $\mathrm{V}$ & 5.92 & 0.6954 & $\mathrm{x}$ & $\begin{array}{l}|H-3 \rightarrow L\rangle(0.2191) \\
|H \rightarrow L+2 ; H-4 \rightarrow L\rangle(0.2191)\end{array}$ \\
\hline VI & 6.15 & 0.6819 & $\mathrm{y}$ & $|H \rightarrow L ; H-3 \rightarrow L+1\rangle(0.2526)$ \\
\hline VII & 6.508 & 0.1217 & $\mathrm{z}$ & $\begin{array}{l}|H-1 \rightarrow L+2\rangle(0.7131) \\
|H-1 \rightarrow L\rangle(0.2104) \\
|H \rightarrow L+2 ; H \rightarrow L+2\rangle(0.2030)\end{array}$ \\
\hline VIII & 6.858 & 1.1338 & $\mathrm{x}$ & $\begin{array}{l}|H \rightarrow L ; H \rightarrow L+10\rangle(0.6912) \\
|H \rightarrow L+2 ; H \rightarrow L+10\rangle(0.3415) \\
|H \rightarrow L+18 ; H \rightarrow L\rangle(0.2759) \\
|H \rightarrow L ; H \rightarrow L+5\rangle(0.4169) \\
|H \rightarrow L+2 ; H \rightarrow L+5\rangle(0.3770) \\
|H \rightarrow L+3 ; H-1 \rightarrow L\rangle(0.2403) \\
|H-1 \rightarrow L+1\rangle(0.2156)\end{array}$ \\
\hline IX & 7.80 & 0.5231 & $\mathrm{z}$ & $\begin{array}{l}|H-1 \rightarrow L ; H-1 \rightarrow L+2\rangle(0.3386) \\
|H \rightarrow L ; H-4 \rightarrow L+2\rangle(0.3164) \\
|H \rightarrow L ; H-4 \rightarrow L+2\rangle(0.2987) \\
|H-1 \rightarrow L ; H-1 \rightarrow L\rangle(0.2722) \\
|H \rightarrow L+1 ; H-1 \rightarrow L+3\rangle(0.2174)\end{array}$ \\
\hline
\end{tabular}

Note: ${ }^{a}$ GS does not correspond to any peak, instead it corresponds to the ground state wavefunction of the isomer. 
Table 10. Excitation energies, $E$, and many-particle wave functions of excited states corresponding to the peaks in the linear absorption spectrum of pentagon $\mathrm{B}_{5}$ ( $c f$. Fig. 12), along with oscillator strength of transition $\left(f_{12}=\frac{2}{3} \frac{m_{e}}{\hbar^{2}}\left(E_{2}-E_{1}\right) \sum_{i}\left|\left\langle m\left|d_{i}\right| G\right\rangle\right|^{2}\right)$, where, $|m\rangle$ denotes the excited state in question, $|G\rangle$, the ground state, and $d_{i}$ is the $i$-th Cartesian component of the electric dipole operator. The polarization in-plane corresponds to the absorption due to light polarized in the plane of isomer. In the wave function, the bracketed numbers are the CI coefficients of a given electronic configuration. Symbols $H$ and $L$ denote HOMO and LUMO orbitals respectively. $H F$ denotes the Hartree-Fock configuration.

\begin{tabular}{|c|c|c|c|c|}
\hline Peak & $E(\mathrm{eV})$ & $f_{12}$ & Polarization & Wave Function \\
\hline $\mathrm{GS}^{a}$ & & & & $\begin{array}{l}|H F\rangle(0.8541) \\
|H-1 \rightarrow L\rangle(0.1906)\end{array}$ \\
\hline I & 1.394 & 0.1805 & $\perp$ to the plane & $\begin{array}{l}|H \rightarrow L+1\rangle(0.8536) \\
|H \rightarrow L+11\rangle(0.1779)\end{array}$ \\
\hline II & 1.818 & 0.0734 & in-plane & $\begin{array}{l}|H \rightarrow L\rangle(0.6524) \\
|H-1 \rightarrow H\rangle(0.4900) \\
|H \rightarrow L+3\rangle(0.2218)\end{array}$ \\
\hline III & 3.504 & 0.6466 & in-plane & $\begin{array}{l}|H-2 \rightarrow L+1\rangle(0.5804) \\
|H-1 \rightarrow H\rangle(0.5131) \\
|H \rightarrow L\rangle(0.3441)\end{array}$ \\
\hline IV & 3.868 & 0.2839 & in-plane & $\begin{array}{l}|H-2 \rightarrow L+1\rangle(0.7968) \\
|H-1 \rightarrow H\rangle(0.3416)\end{array}$ \\
\hline $\mathrm{V}$ & 4.379 & 0.1885 & in-plane & $\begin{array}{l}|H-3 \rightarrow H\rangle(0.8185) \\
|H-4 \rightarrow L\rangle(0.1900)\end{array}$ \\
\hline VI & 5.576 & 5.0600 & in-plane & $\begin{array}{l}|H-1 \rightarrow L\rangle(0.7309) \\
|H-4 \rightarrow L\rangle(0.3583) \\
|H-1 \rightarrow L+3\rangle(0.1994) \\
|H-3 \rightarrow H\rangle(0.1808) \\
|H-2 \rightarrow L+1\rangle(0.4415) \\
|H-3 \rightarrow L\rangle(0.4045) \\
|H-4 \rightarrow H\rangle(0.4000) \\
|H \rightarrow L\rangle(0.2399) \\
|H-1 \rightarrow L+4\rangle(0.2039)\end{array}$ \\
\hline
\end{tabular}

Note: ${ }^{a}$ GS does not correspond to any peak, instead it corresponds to the ground state wavefunction of the isomer. 
Table 11. (Continued from last page) Excitation energies, E, and many-particle wave functions of excited states corresponding to the peaks in the linear absorption spectrum of pentagon $\mathrm{B}_{5}$ ( $c f$. Fig. 12), along with oscillator strength of transition $\left(f_{12}=\frac{2}{3} \frac{m_{e}}{\hbar^{2}}\left(E_{2}-E_{1}\right) \sum_{i}\left|\left\langle m\left|d_{i}\right| G\right\rangle\right|^{2}\right)$, where, $|m\rangle$ denotes the excited state in question, $|G\rangle$, the ground state, and $d_{i}$ is the $i$-th Cartesian component of the electric dipole operator. The polarization in-plane corresponds to the absorption due to light polarized in the plane of isomer. In the wave function, the bracketed numbers are the CI coefficients of a given electronic configuration. Symbols $H$ and $L$ denote HOMO and LUMO orbitals respectively. $H F$ denotes the Hartree-Fock configuration.

\begin{tabular}{|c|c|c|c|c|}
\hline Peak & $E(\mathrm{eV})$ & $f_{12}$ & Polarization & Wave Function \\
\hline \multirow[t]{11}{*}{ VII } & 6.305 & 4.9168 & in-plane & $|H-3 \rightarrow L\rangle(0.4073)$ \\
\hline & & & & $|H-1 \rightarrow L+4\rangle(0.3328)$ \\
\hline & & & & $|H-1 \rightarrow L+9\rangle(0.2952)$ \\
\hline & & & & $|H-2 \rightarrow L+1\rangle(0.2870)$ \\
\hline & & & & $|H-4 \rightarrow L+4\rangle(0.2772)$ \\
\hline & & & & $|H-4 \rightarrow L+9\rangle(0.2531)$ \\
\hline & 6.528 & 0.7269 & in-plane & $|H-1 \rightarrow L\rangle(0.5596)$ \\
\hline & & & & $|H-4 \rightarrow L\rangle(0.3400)$ \\
\hline & & & & $|H-2 \rightarrow L+2\rangle(0.2761)$ \\
\hline & & & & $|H-3 \rightarrow L+4\rangle(0.2667)$ \\
\hline & & & & $|H-3 \rightarrow L+9\rangle(0.2598)$ \\
\hline \multirow[t]{8}{*}{ VIII } & 7.161 & 1.9516 & in-plane & $|H-4 \rightarrow L+4\rangle(0.4305)$ \\
\hline & & & & $|H-4 \rightarrow L+9\rangle(0.3859)$ \\
\hline & & & & $|H-3 \rightarrow L\rangle(0.3776)$ \\
\hline & & & & $|H-1 \rightarrow L+4\rangle(0.2487)$ \\
\hline & 7.283 & 0.5947 & in-plane & $|H \rightarrow L+6\rangle(0.7102)$ \\
\hline & & & & $|H \rightarrow L+9\rangle(0.3359)$ \\
\hline & & & & $|H-3 \rightarrow L+4\rangle(0.2091)$ \\
\hline & & & & $|H-3 \rightarrow L+9\rangle(0.1854)$ \\
\hline \multirow[t]{4}{*}{ IX } & 7.702 & 1.0247 & $\perp$ to the plane & $|H \rightarrow L+11\rangle(0.7980)$ \\
\hline & & & & $|H \rightarrow L+14\rangle(0.2692)$ \\
\hline & 7.750 & 0.8764 & in-plane & $|H-1 \rightarrow L+3\rangle(0.8332)$ \\
\hline & & & & $|H-1 \rightarrow L+19\rangle(0.1555)$ \\
\hline
\end{tabular}


Table 12. Excitation energies, $E$, and many-particle wave functions of excited states corresponding to the peaks in the linear absorption spectrum of distorted triangular-bipyramid $\mathrm{B}_{5}$ ( $c f$. Fig. 13), along with oscillator strength of transition $\left(f_{12}=\frac{2}{3} \frac{m_{e}}{\hbar^{2}}\left(E_{2}-E_{1}\right) \sum_{i}\left|\left\langle m\left|d_{i}\right| G\right\rangle\right|^{2}\right)$, where, $|m\rangle$ denotes the excited state in question, $|G\rangle$, the ground state, and $d_{i}$ is the $i$-th Cartesian component of the electric dipole operator. The polarization $x, y$ and $z$ corresponds to the absorption due to light polarized along $x-, y-$ and $z$ - axis respectively. In the wave function, the bracketed numbers are the CI coefficients of a given electronic configuration. Symbols $H$ and $L$ denote HOMO and LUMO orbitals respectively. $H F$ denotes the Hartree-Fock configuration.

\begin{tabular}{|c|c|c|c|c|}
\hline Peak & $E(\mathrm{eV})$ & $f_{12}$ & Polarization & Wave Function \\
\hline $\mathrm{GS}^{a}$ & & & & $\begin{array}{l}|H F\rangle(0.8615) \\
|H-1 \rightarrow H\rangle(0.2165) \\
|H-1 \rightarrow H ; H-1 \rightarrow L\rangle(0.1371) \\
|H-2 \rightarrow H ; H-1 \rightarrow L\rangle(0.1143)\end{array}$ \\
\hline I & 1.774 & 0.4172 & $\mathrm{z}$ & $|H-1 \rightarrow L+4\rangle(0.8689)$ \\
\hline II & 3.161 & 0.5817 & $\mathrm{y}$ & $\begin{array}{l}|H-2 \rightarrow H\rangle(0.8734) \\
|H-1 \rightarrow H ; H-1 \rightarrow L\rangle(0.2303)\end{array}$ \\
\hline III & 4.274 & 0.3879 & $\mathrm{z}$ & $\begin{array}{l}|H-1 \rightarrow L+2\rangle(0.8666) \\
|H-1 \rightarrow L+6\rangle(0.1775)\end{array}$ \\
\hline IV & 5.487 & 1.4856 & $\mathrm{y}$ & $\begin{array}{l}|H-1 \rightarrow H ; H-1 \rightarrow L\rangle(0.7812) \\
|H-2 \rightarrow H\rangle(0.2336) \\
|H-2 \rightarrow H ; H-1 \rightarrow L\rangle(0.2332)\end{array}$ \\
\hline $\mathrm{V}$ & 6.408 & 0.8914 & $\mathrm{z}$ & $\begin{array}{l}|H-4 \rightarrow H\rangle(0.7837) \\
|H-2 \rightarrow L+2\rangle(0.3357) \\
|H-2 \rightarrow H ; H-1 \rightarrow L+1\rangle(0.2274)\end{array}$ \\
\hline VI & 7.519 & 3.4471 & $\mathrm{x}$ & $\begin{array}{l}|H-2 \rightarrow H ; H-1 \rightarrow L+2\rangle(0.8133) \\
|H-2 \rightarrow H ; H-1 \rightarrow L+6\rangle(0.2669)\end{array}$ \\
\hline VII & 7.744 & 0.1349 & $\mathrm{x}$ & $\begin{array}{l}|H-1 \rightarrow L ; H-1 \rightarrow L+1\rangle(0.6105) \\
|H-1 \rightarrow L+4\rangle(0.4305) \\
|H-2 \rightarrow L+1\rangle(0.2271)\end{array}$ \\
\hline
\end{tabular}

Note: ${ }^{a}$ GS does not correspond to any peak, instead it corresponds to the ground state wavefunction of the isomer. 


\section{Bibliography}

1. J. A. Alonso, Structure and Properties of Atomic Nanostructures, Imperial College Press, (2005), London.

2. T. Kondow, Y, Kawazoe and K, Ohno, Clusters and Nanomaterials, Springer-Verlag, (2002).

3. J. Jellinek, Theory of Atomic and Molecular Clusters - With a Glimpse at Experiments, Springer-Verlag, (1999).

4. W. A. de Heer, The physics of simple metal clusters: experimental aspects and simple models, Rev. Mod. Phys., 65, 611 (1993).

5. J. Plešek, Potential applications of boron cluster compounds., Chem. Rev., 92, 269 (1992).

6. R. H. Xie, G. W. Bryant, J. Zhao, T. Kar, and V. H. Smith, Tunable optical properties of icosahedral, dodecahedral, and tetrahedral clusters, Phys. Rev. B, 71, 125422 (2005).

7. N. S. Goroff, Mechanism of fullerene formation, Acc. Chem. Res., 29, 77 (1996).

8. T. Belz, F. Z. H. Werner, M. W. U. Klengler, E. Z. B. Tesche, A. Reller, and Robert Schlögl, On the mechanism of fullerene formation, Angew. Chem. Int. Ed., 33, 1866 (2003).

9. R. N. Grimes, Boron clusters come of age, J. Chem. Educ., 81, 657 (2004).

10. Y. Zhao, Y. H. Kim, A.C. Dillon, M. J. Heben, and S. B. Zhang, Hydrogen storage in novel organometallic buckyballs, Phys. Rev. Lett., 94, 155504 (2005).

11. I. Cabria, M. J. López, and J. A. Alonso, Density functional calculations of hydrogen adsorption on boron nanotubes and boron sheets, Nanotechnology, 17, 778 (2006).

12. S. A. Shevlin and Z. X. Guo, Hydrogen sorption 
in defective hexagonal $B_{n}$ sheets and $B_{n}$ nanotubes, Phys. Rev. B, 76, 024104 (2007).

13. H. J. Zhai, B. Kiran, J. Li, and L. S. Wang, Hydrocarbon analogues of boron clusters - planarity, aromaticity and antiaromaticity, Nature Materials, 2, 827 (2003).

14. L. Hanley, J. L. Whitten, and S. L. Anderson, Collision-induced dissociation and ab initio studies of boron cluster ions: determination of structures and stabilities J. Phys. Chem., 92, (20), 5803 (1988).

15. H. J. Zhai, A. N. Alexandrova, K. A. Birch, A. I. Boldyrev, and L. S. Wang, Hepta- and octacoordinate boron in molecular wheels of eight-and nineatom boron clusters: Observation and confirmation, Angew. Chem. Int. Ed., 42, 6004 (2003).

16. C. Romanescu, T. R. Galeev, W. L. Li, A. I. Boldyrev, and L. S. Wang, Aromatic metal-centered monocyclic boron rings: $C o\left(B_{8}^{-}\right.$and $R u\left(B_{9}^{-}\right.$, Angew. Chem. Int. Ed., 50, (40), 9334 (2011).

17. B. Kiran, S. Bulusu, H. J. Zhai, S. Yoo, X. C. Zeng, and L. S. Wang Planar-to-tubular structural transition in boron clusters: $B_{20}$ as the embryo of singlewalled boron nanotubes, Proc. Nat. Acad. Sci. USA, $102,(4), 961$ (2005).

18. S. J. La Placa, P. A. Roland, and J. J. Wynne, Boron clusters (bn, $n=2-52)$ produced by laser ablation of hexagonal boron nitride, Chem. Phys. Lett., 190, (34), 163 (1992).

19. S. Sahu and A. Shukla, Probing aromaticity of borozene through optical and dielectric response: a theoretical study, Nano. Res. Lett, 5, (4), 714 (2010).

20. J. S. Lauret, R. Arenal, F. Ducastelle, A. Loiseau, M. Cau, B. Attal-Tretout, E. Rosencher, and L. Goux-Capes, Optical transitions in single-wall boron nitride nanotubes, Phys. Rev. Lett., 94, 037405 (2005).

21. N. G. Szwacki, A. Sadrzadeh, and B. I. Yakobson, $\mathrm{B}_{80}$ fullerene: An Ab Initio prediction of geometry, stability, and electronic structure, Phys. Rev. Lett., 98, 166804 (2007).

22. S. Chacko, D. G. Kanhere, and I. Boustani, Ab initio density functional investigation of $\mathrm{B}_{24}$ clusters: rings, tubes, planes, and cages, Phys. Rev. B, 68, 035414, (2003).

23. A. Abdurahman, A. Shukla, and G. Seifert, Ab initio many-body calculations of static dipole polarizabilities of linear carbon chains and chainlike boron clusters, Phys. Rev. B, 66, 155423 (2002).

24. M. P. Johansson, On the strong ring currents in b20 and neighboring boron toroids, J. Phys. Chem. C, 113, (2), 524 (2009).

25. D. E. Bean and P. W. Fowler, Double aromaticity in boron toroids, J. Phys. Chem. C, 113, (35), 15569 (2009).

26. S. R. Langhoff and C. W. Bauschlicher, Theoretical study of the spectroscopy of $B_{2}, J$. Chem. Phys., 95,
(8), $5882(1991)$

27. P. J. Bruna and J. S. Wright, Strongly bound multiply excited states of $B^{+2}$ and $B_{2}, J$. Chem. Phys., 91, (2), 1126 (1989).

28. I. A. Howard and A. K. Ray, A correlation study of boron dimers and trimers, Z. Phy. D., 42, 299 (1997).

29. I. Boustani, Systematic ab initio investigation of bare boron clusters: Determination of the geometry and electronic structures of boron clusters, Phys. Rev. B, 55, 16426 (1997).

30. J. Niu, B. K. Rao, and P. Jena, Atomic and electronic structures of neutral and charged boron and boron-rich clusters, J. Chem. Phys., 107, (1), 132 (1997).

31. M. Atiş, C. Özdogan, and Z. B. Güvenç, Structure and energetic of $B_{n}(n=2-12)$ clusters- electronic structure calculations, Int. J. Quantum Chem., 107, (3), 729 (2007).

32. J. I. Aihara, H. Kanno, and T. Ishida, Aromaticity of planar boron clusters confirmed, J. Am. Chem. Soc., 127, (38), 13324 (2005).

33. M. Saito and Y. Miyamoto, Theoretical identification of the smallest fullerene, $C_{20}$ Phys. Rev. Lett., 87, 035503 (2001).

34. C. R. C. Wang, S. Pollack, D. Cameron, and M. M. Kappes, Optical absorption spectroscopy of sodium clusters as measured by collinear molecular beam photodepletion, J. Chem. Phys., 93, (6), 3787 (1990).

35. J. Bowlan, A. Liang, and W. A. de Heer, How metallic are small sodium clusters? , Phys. Rev. Lett., 106, 043401 (2011).

36. G. Pal, Y. Pavlyukh, W. Hübner, and H. C. Schneider, Optical absorption spectra of finite systems from a conserving bethe-salpeter equation approach Eur. Phys. J. B, 79, (3), 327 (2011).

37. I. Boustani, W. Pewestorf, P. Fantucci, V. B. Koutecký, and J. Koutecký, Systematic ab initio configuration-interaction study of alkali-metal clusters: Relation between electronic structure and geometry of small li clusters, Phys. Rev. B, 35, 9437 (1987).

38. J. M. Pacheco and J. L. Martins, Ab initio pseudopotential calculation of the photo-response of metal clusters, J. Chem. Phys., 106, (14), 6039 (1997).

39. M. A. L. Marques and S. Botti, The planar-totubular structural transition in boron clusters from optical absorption, J. Chem. Phys., 123, (1), 014310 (2005).

40. S. Botti, A. Castro, N. N. Lathiotakis, X. Andrade, and M. A. L. Marques, Optical and magnetic properties of boron fullerenes, Phys. Chem. Chem. Phys., 11, (22), 4523 (2009).

41. M. W. Schmidt, K. K. Baldridge, J. A. Boatz, S. T. Elbert, M. S. Gordon, J. H. Jensen, S. Koseki, 
N. Matsunaga, K. A. Nguyen, S. Su, T. L. Windus, M. Dupuis, and J. A. Montgomery. General atomic and molecular electronic structure system, J. Comput. Chem., 14, (11), 1347 (1993).

42. L. E. McMurchie, S. T. Elbert, S. R. Langhoff, and E. R. Davidson, MELD package from Indiana University. It has been modified by us to handle bigger systems.

43. P. Sony and A. Shukla, Large-scale correlated study of excited state absorptions in naphthalene and anthracene, J. Chem. Phys., 131, (1), 014302 (2009).

44. P. Sony and A. Shukla, Large-scale correlated calculations of linear optical absorption and low-lying excited states of polyacenes: Pariser-parr-pople hamiltonian, Phys. Rev. B, 75, 155208 (2007).

45. P. Sony and A. Shukla, Photoinduced absorption in disubstituted polyacetylenes: Comparison of theory with experiments, Phys. Rev. B, 71, 165204 (2005).

46. A. Shukla, Correlated theory of triplet photoinduced absorption in phenylene-vinylene chains, Phys. Rev. $B, \mathbf{6 5}, 125204$ (2002).

47. J. Blanc, V. B. Koutecký, M. Broyer, J. Chevaleyre, P. Dugourd, J. Koutecký, C. Scheuch, J. P. Wolf, and L. Wöste. Evolution of the electronic structure of lithium clusters between four and eight atoms., J. Chem. Phys., 96, (3),1793 (1992).

48. G. Herzberg. Molecular Spectra and Molecular Structure: Constants of diatomic Molecules, Van Nostrand, (1979).

49. K. L. Schuchardt, B. T. Didier, T. Elsethagen, L. Sun, V. Gurumoorthi, J. Chase, J. Li, and T. L. Windus. Basis set exchange : a community database for computational sciences, J. Chem. Inf. Model., 47, (3), 1045 (2007).

50. David Feller, The role of databases in support of computational chemistry calculations, J. Comput. Chem., 17, (13), 1571 (1996). 